\title{
Edge flame propagation statistics in igniting mono-disperse droplet-laden mixtures
}

V.S. Papapostolou, ${ }^{1,}$ a) C. Turquand d'Auzay, ${ }^{1}$ G. Ozel Erol, ${ }^{1}$ and N. Chakraborty ${ }^{1}$ School of Engineering, Newcastle University, Newcastle-Upon-Tyne NE1 7RU, United Kingdom 
The effects of droplet diameter, overall (i.e. liquid+gaseous phases) equivalence ratio and turbulence intensity on the edge flame propagation statistics for localised forced ignition of uniformly dispersed n-heptane droplet-laden mixtures under homogeneous isotropic decaying turbulence have been analysed based on Direct Numerical Simulations (DNS) data. It has been found that the edge flame structure becomes increasingly prominent for large overall equivalence ratios and droplet diameters. Although the mean edge flame speed has been found to be positive and its most probable value remains comparable to the theoretical value for laminar edge flames in purely gaseous mixtures, the mean values have been found to decrease and the probabilities of finding locally negative edge flame speeds have been found to increase with increasing turbulence intensity. The marginal probability density function, and curvature and strain rate dependences of the edge flame speed have been found to be principally governed by the displacement speed of the fuel mass fraction isosurface intersecting the stoichiometric mixture fraction isosurface. The displacement speed of the stoichiometric mixture fraction isosurface has also been found to influence the local scalar gradient dependences of the edge flame speed in this configuration, especially for large droplets. The displacement speed of the fuel mass fraction isosurface $S_{d}$ has been found to be principally governed by leading order contributions of the reaction and molecular diffusion components and the evaporation contribution remains weak in comparison to these leading order contributors. The local edge flame speed exhibits non-linear curvature and strain rate dependences and its variation with the magnitudes of both fuel mass fraction and mixture fraction gradients has been found to be non-monotonic for all cases considered here. The correlations of the edge flame speed with curvature, strain rate and scalar gradient have been found to be qualitatively similar to the corresponding statistics reported in the existing literature for edge flames in purely gaseous mixtures. Additionally, the curvature and tangential strain rate dependences of edge flame speed have been found to be dependent on the droplet size and overall equivalence ratio, and these dependences become weak for cases with large droplets.

Keywords: Edge flame, droplet-laden mixtures, overall equivalence ratio, turbulence intensity, Direct Numerical Simulations 
a)Electronic mail: V.S.Papapostolu1@newcastle.ac.uk 


\section{INTRODUCTION}

In many modern combustion devices (e.g. automotive engines, gas turbines, etc.), chemical reactions take place in the presence of inhomogeneous mixtures where both the fuel and oxidiser are neither homogeneously mixed nor totally segregated from each other. This situation is prevalent in the case of droplet combustion. Localised forced ignition (e.g. spark ignition, laser ignition etc.) of droplet-laden turbulent flows has applications in Direct Injection engines along with high-altitude relight in aero gas turbine applications. Flames resulting from localised ignition of inhomogeneous mixtures often exhibit edge flame structure as shown in previous numerical studies ${ }^{1 / 6}$. The edge flame propagation plays a pivotal role in flame stabilisation and flame extinction $\frac{78}{78}$ and it has been analysed extensively for

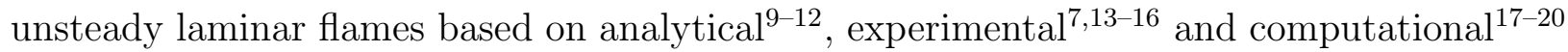
studies. A relatively limited number of studies $21 \sqrt{26}$ analysed edge flame propagation based on turbulent mixing layers and turbulent jet flows. Most of the aforementioned analyses $\frac{1}{421} 26$ on turbulent edge flame propagation have been conducted for gaseous phase combustion and relatively limited effort has been directed to the edge flame propagation in the context of droplet combustion ${ }^{5}$. Moreover, the majority of computational studies on edge flame propagation focused upon the effects of curvature, strain rate and scalar gradient dependences of the density-weighted displacement speed of a fuel mass fraction isosurface ${ }^{1 / 6] 17}[20$ which intersects with the stoichiometric mixture fraction isosurface. However, in addition to the displacement speed of the fuel mass fraction isosurface, the displacement speed of the mixture fraction isosurface also affects the speed with which the flame propagates on the stoichiometric mixture fraction isosurface $24 \sqrt{26}$. To the best of the authors' knowledge, there have been limited analyses $27 / 28$, which concentrated on edge flame speed statistics in droplet-laden mixtures. Semi-analytical studies ${ }^{27 / 28}$ have highlighted the importance of the droplet diameter distribution and reactants diffusivities on the edge flame speed under laminar droplet laden mixing layers. However, there is yet to be a detailed investigation of edge flame propagation in turbulent droplet-laden mixtures and this void has been addressed here by carrying out a Direct Numerical Simulation (DNS) analysis, which concentrated on edge flame speed statistics in igniting droplet-laden turbulent mixtures. Thus, three-dimensional DNS of localised forced ignition for n-heptane droplets under decaying homogeneous isotropic turbulence has been carried out for uniformly dispersed mono-sized droplets for different initial diameters 
and overall (i.e. liquid+gaseous phases) equivalence ratios. The overall equivalence ratio (i.e. fuel to air ratio by mass normalised by fuel to air ratio under stoichiometric condition) is calculated by summing the total amount of the fuel present in both liquid and gaseous phases, (i.e. $\phi_{o v}=\phi_{l}+\phi_{g}$ with subscripts 1 and g stand for liquid and droplet phases, respectively). Under the initial conditions considered in this study, all the fuel is presented in the form of liquid droplets, and thus the total number of droplets initially present in the domain determines the initial overall equivalence ratio.

The aims of this paper are:

(i) To demonstrate the effects that droplet size and the overall equivalence ratio (or number density of droplets) have on the edge flame speed and its various components.

(ii) To analyse and explain the influences of droplet size and overall equivalence ratio on the curvature, scalar gradient and strain rate dependences of edge flame speed.

(iii) To compare the statistics of edge flame propagation in droplet-laden mixtures with previously obtained results for edge flames in purely gaseous mixtures, and indicate the modelling implications of these findings.

The rest of the paper will take the following form. The information related to mathematical background and numerical implementation will be provided in the next two sections. The results will be presented in Section 4 along with its discussion. The summary of the main findings and conclusions are provided in the final section of this paper.

\section{MATHEMATICAL BACKGROUND}

Three-dimensional DNS simulations have been carried out for 27 different parameter sets, consisting of three different initial turbulence intensities, overall equivalence ratios and droplet diameters. Thus, a modified single-step Arrhenius type irreversible chemical reaction is chosen for the current analysis to keep the computational cost of the study within reasonable limits. This chemical mechanism is expressed as:

$$
\text { Fuel }+s \cdot \text { Oxidiser } \rightarrow(1+s) \text { Products }
$$

where $s$ is the mass of oxygen consumed per unit mass of fuel consumption. The chemical mechanism was implemented following Tarrazo et al. $\stackrel{29}{ }$, where the activation energy, $E_{a c}$, and 
the heat of combustion have been taken to be functions of the gaseous equivalence ratio, $\phi_{g}$, which provides a realistic equivalence ratio dependence for the unstrained laminar burning velocity $S_{L_{\phi_{g}}}$ in hydrocarbon-air flames. A similar methodology has been implemented by Haruki et al.$^{30}$ for a two-step mechanism in a DNS study of spray flames. For simplicity, in the present study the Lewis number $L e_{i}=\alpha_{i} / D_{i}$ (where $\alpha_{i}$ is the thermal diffusivity and $D_{i}$ is the species diffusivity of the $i^{\text {th }}$ species) of all species is taken to be equal to unity. All species in the gaseous phase are taken to be ideal gases, and thus for the compressible flow DNS, as is the case here, the ideal gas law applies. Standard values were taken for the ratio of specific heats $\gamma\left(\gamma=C_{p}^{g} / C_{v}^{g}=1.4\right.$, where $C_{p}^{g}$ and $C_{v}^{g}$ are the gaseous specific heats at constant pressure and volume, respectively) and Prandtl number $\left(\operatorname{Pr}=\mu C_{p}^{g} / \lambda=0.7\right.$, where $\mu$ is the dynamic viscosity and $\lambda$ is the thermal conductivity of the gaseous phase). Each individual droplet is tracked in a Lagrangian sense, whilst the compressible Navier-Stokes equations are solved in the Eulerian frame for the carrier gaseous phase. The evolution of the droplet related quantities such as the position, $\overrightarrow{x_{d}}$, velocity, $\overrightarrow{u_{d}}$, diameter $a_{d}$ and temperature $T_{d}$ are expressed following the work of Reveillon and Vervisch $\frac{31}{}$. The transport equations of the position, $\overrightarrow{x_{d}}$, velocity, $\overrightarrow{u_{d}}$, diameter, $a_{d}$ and temperature, $T_{d}$, of the droplets indicated by the subscript $d$ are given by ${ }^{5 / 31 \mid 32}$ :

$$
\begin{aligned}
\frac{d \overrightarrow{x_{d}}}{d t} & =\overrightarrow{u_{d}}, \\
\frac{d \overrightarrow{u_{d}}}{d t} & =\frac{\vec{u}\left(\overrightarrow{x_{d}}, t\right)-\overrightarrow{u_{d}}}{\tau_{d}^{u}}, \\
\frac{d a_{d}^{2}}{d t} & =-\frac{a_{d}^{2}}{\tau_{d}^{p}}, \\
\frac{d T_{d}}{d t} & =\frac{\hat{T}\left(\overrightarrow{x_{d}}, t\right)-T_{d}-B_{d} L_{v} / C_{p}^{g}}{\tau_{d}^{T}}
\end{aligned}
$$

where $\hat{T}$ is the instantaneous dimensional temperature of the gaseous phase, $L_{v}$ is the latent heat of vaporisation, and $\tau_{d}^{u}, \tau_{d}^{p}$ and $\tau_{d}^{T}$ are relaxation timescales for velocity, droplet diameter and temperature, respectively. They are defined as ${ }^{5131 / 32}$ :

$$
\begin{aligned}
\tau_{d}^{u} & =\frac{\rho_{d} a_{d}^{2}}{18 C_{u} \mu} \\
\tau_{d}^{p} & =\frac{\rho_{d} a_{d}^{2} S c}{4 \mu S h_{c} \ln \left(1+B_{d}\right)} \\
\tau_{d}^{T} & =\frac{\rho_{d} a_{d}^{2} \operatorname{Pr} B_{d} C_{p}^{L}}{6 \mu N u_{c} \ln \left(1+B_{d}\right) C_{p}^{g}}
\end{aligned}
$$


Here, $\rho_{d}$ is the droplet density, $S c$ is the Schmidt number which is taken to be 0.7 (i.e. $S c=$ 0.7 ) following Refs. ${ }^{30+32}, C_{p}^{L}$ is the specific heat for the liquid phase, $C_{u}=1+\operatorname{Re}_{d}^{2 / 3} / 6$ is the correction for the drag coefficient with $R e_{d}$ being the droplet Reynolds number, $B_{d}$ is the Spalding number, $S h_{c}$ is the corrected Sherwood number and $N u_{c}$ is the corrected Nusselt number, which are defined as 513132 :

$$
\begin{aligned}
R e_{d} & =\frac{\rho\left|\vec{u}\left(\overrightarrow{x_{d}}, t\right)-\overrightarrow{u_{d}}\right| a_{d}}{\mu} \\
B_{d} & =\frac{Y_{F}^{s}-Y_{F}\left(\overrightarrow{x_{d}}, t\right)}{1-Y_{F}^{s}} \\
S h_{c} & =N u_{c}=2+\frac{0.555 R e_{d} S c}{\left(1.232+R e_{d} S c^{4 / 3}\right)^{1 / 2}}
\end{aligned}
$$

where $\rho$ is the gas density, while the fuel mass fraction $Y_{F}$ at the surface of the $\operatorname{droplet}\left(Y_{F}^{s}\right)$ and the partial pressure of the fuel vapour at the droplet surface $p_{F}^{s}$ are expressed by ${ }^{5131322}$ :

$$
\begin{aligned}
& p_{F}^{s}=p_{\text {ref }} \exp \left(L_{v}\left[\frac{1}{R T_{\text {ref }}^{s}}-\frac{1}{R T_{d}^{s}}\right]\right) \\
& Y_{F}^{s}=\left(1+\frac{W_{\text {air }}}{W_{F}}\left[\frac{p\left(\overrightarrow{x_{d}}, t\right)}{p_{F}^{s}}-1\right]\right)
\end{aligned}
$$

In Eq. 12, $T_{r e f}^{s}$ denotes the boiling point of the fuel at a pressure $p_{r e f}, R$ is the specific gas constant, and the temperature at the droplet surface $T_{d}^{s}$ is assumed to be $T_{d}$, and $W_{\text {air }}$ and $W_{F}$ are the molecular weights of air and fuel, respectively. The coupling between the Lagrangian and Eulerian phases is obtained through additional source terms in the gaseous phase transport equations ${ }^{5131132}$ :

$$
\frac{\partial \rho \psi}{\partial t}+\frac{\partial \rho u_{j} \psi}{\partial x_{j}}=\frac{\partial}{\partial x_{j}}\left(\Gamma_{\psi} \frac{\partial \psi_{1}}{\partial x_{j}}\right)+\dot{\omega}_{\psi}+\dot{S}_{g}+\dot{S}_{\psi}
$$

where $\psi=\left\{1, u_{i}, e, Y_{F}, Y_{O}\right\}$ and $\psi_{1}=\left\{1, u_{i}, \hat{T}, Y_{F}, Y_{O}\right\}$ for the conservation equations of mass, momentum, internal energy (e), fuel and oxidiser mass fractions (i.e. $Y_{F}$ and $Y_{O}$ ), respectively, where $\hat{T}$ is the dimensional temperature. For $\psi=\left\{1, u_{i}, e, Y_{F}, Y_{O}\right\}$, the diffusion coefficient is given by $\Gamma_{\psi}=\rho \nu / \sigma_{\psi}$, whereas for $\psi=e=\int_{T_{r e f}}^{\hat{T}} C_{v}^{g} d T^{\prime}+u_{k} u_{k} / 2$, the diffusion coefficient is taken to be the thermal conductivity (i.e. $\Gamma_{\psi}=\lambda$ ) with $u_{i}$ being the velocity component in the $i^{t h}$ direction. The $\dot{\omega}_{\psi}$ term in eq. 14 arises due to the chemical reaction rate, which only appears in the conservation equations of energy and species. The term $\dot{S}_{g}$ for $\psi=u_{i}$ is given by $\dot{S}_{g}=-\partial p / \partial x_{i}$ where $p$ is the local pressure. Similarly, $\dot{S}_{g}$ for $\psi=e$ is given by: $\dot{S}_{g}=-\partial\left(u_{k} p\right) / \partial x_{k}+\partial\left(\tau_{k i} u_{i}\right) / \partial x_{k}-\partial\left[\rho \Sigma_{k=1}^{N} h_{s, k} Y_{k} V_{k, i}\right] / \partial x_{i}+q^{\prime \prime \prime}$ where $V_{k, i}$ is 
the diffusion velocity of the $\mathrm{k}^{\text {th }}$ species in the $\mathrm{i}^{\text {th }}$ direction, $h_{s, k}$ is the sensible enthalpy of the $\mathrm{k}^{\text {th }}$ species and $q^{\prime \prime \prime}$ is the energy source term due to external energy addition. The term $\dot{S}_{g}$ appears only in the conservation equations of momentum and energy and this term does not appear in the conservation equations of mass and species (i.e. for $\psi=1, Y_{F}, Y_{O}$ ). The $\dot{S}_{\psi}$ term in eq. 14 , is the appropriate source term due to the coupling between Eulerian and Lagrangian phases, which is tri-linearly interpolated from the droplets sub grid position, $\overrightarrow{x_{d}}$, to the eight surrounding nodes, whilst $\nu$ represents the kinematic viscosity and $\sigma_{\psi}$ refers to an appropriate Schmidt number corresponding to $\psi$.

In order to account for the effects of the localised forced ignition, an additional source term $\left(q^{\prime \prime \prime}=A_{s p} \exp \left(-r^{2} / 2 R_{s p}^{2}\right)\right.$ with $r$ being the distance from the ignitor centre and $R_{s p}$ representing the characteristic width of energy deposition) is added to the energy conservation equation. It deposits energy following a Gaussian distribution in the radial direction from the ignition centre. The constant $A_{s p}$ is determined by a volume integration which leads to the total ignition power $\dot{Q}^{1 / 613334}$ :

$$
\dot{Q}=\int_{V} q^{\prime \prime \prime} d V=a_{s p} \rho_{0} C_{p} \tau T_{0}\left(\frac{4}{3} \pi \delta_{z}^{3}\right)\left[\frac{\mathcal{H}(t)-\mathcal{H}\left(t-t_{s p}\right)}{t_{s p}}\right]
$$

where $a_{s p}$ is a parameter determining the total energy deposited by the ignitor, $\tau=\left(T_{a d_{\phi_{g}=1}}-\right.$ $\left.T_{0}\right) / T_{0}$ is the heat release parameter, where $T_{a d_{\phi_{g}=1}}$ and $T_{0}$ are the adiabatic flame temperature of the stoichiometric mixture and unburned gas temperature, respectively. The Zel'dovich flame thickness of the stoichiometric mixture, $\delta_{z}$ is defined as $\delta_{z}=\alpha_{T_{0}} / S_{L_{\phi_{g}=1}}$, where $\alpha_{T_{0}}$ is the thermal diffusivity of the unburned gas and $S_{L_{\phi_{g}=1}}$ is the unstrained laminar burning velocity of the stoichiometric mixture. To ensure that the ignitor is only active until $t=t_{s p}$, Heaviside functions are used and defined as $\mathcal{H}(t)$, and $\mathcal{H}\left(t-t_{s p}\right)$. The energy deposition duration $t_{s p}$, is expressed as $t_{s p}=b_{s p} t_{f}$, where $t_{f}=\delta_{z} / S_{L_{\phi_{g}=1}}$ is a characteristic chemical timescale, while the energy deposition parameter $b_{s p}$ is taken to be $b_{s p}=0.2$ in the present study, which falls within its optimal range ${ }^{355}$. In the present study, $a_{s p}, b_{s p}$ and $R_{s p}$ are kept constant (i.e. $a_{s p}=15.0, b_{s p}=0.2, R_{s p}=2.45 \delta_{z}$ ) such that successful ignition and subsequent self-sustained propagation occur for all cases investigated here.

As the present study aims to investigate edge flame propagation for droplet-laden turbulent spray flames, it is necessary to discuss how the edge flame is characterised. Here, the intersection between the stoichiometric mixture fraction (i.e. $\xi=\xi_{s t}$ ) iso-surface with the fuel mass fraction $Y_{F}$ iso-surfaces within a reaction progress variable $c$ range (i.e. $0.1 \leq c \leq 0.9$ ) 
has been considered. The mixture fraction $(\xi)$ and progress variable $(c)$ are defined as:

$$
\begin{aligned}
\xi & =\frac{\left(Y_{F}-Y_{O} / s+Y_{O_{\infty}} / s\right)}{Y_{F_{\infty}}+Y_{O_{\infty}} / s} \\
c & =\frac{\left[(1-\xi) Y_{O_{\infty}}-Y_{O}\right]}{\left[(1-\xi) Y_{O_{\infty}}-\max \left(0,\left(\xi_{s t}-\xi\right) / \xi_{s t}\right) Y_{O_{\infty}}\right]}
\end{aligned}
$$

where $Y_{O}$ is the local oxygen mass fraction, $Y_{O_{\infty}}=0.233$ is the oxygen mass fraction in air, and $Y_{F_{\infty}}=1.0$ is the fuel mass fraction in the pure fuel stream. For n-heptane $\left(\mathrm{C}_{7} \mathrm{H}_{16}\right)$ combustion, $s=3.52$ which leads to a stoichiometric mixture fraction value of $\xi_{s t}=0.0621$.

The edge flame statistics are extracted for the reaction progress variable range, $0.1 \leq c \leq$ 0.9 on the stoichiometric mixture fraction $\left(\xi=\xi_{s t}\right)$ isosurface. In order to define the edge flame speed, it is necessary to create a coordinate system attached to the edge flame. To help with understanding this, a diagram of the coordinate system is shown in Fig. 1, which follows the schematic diagram laid out by Karami et al.24|25.

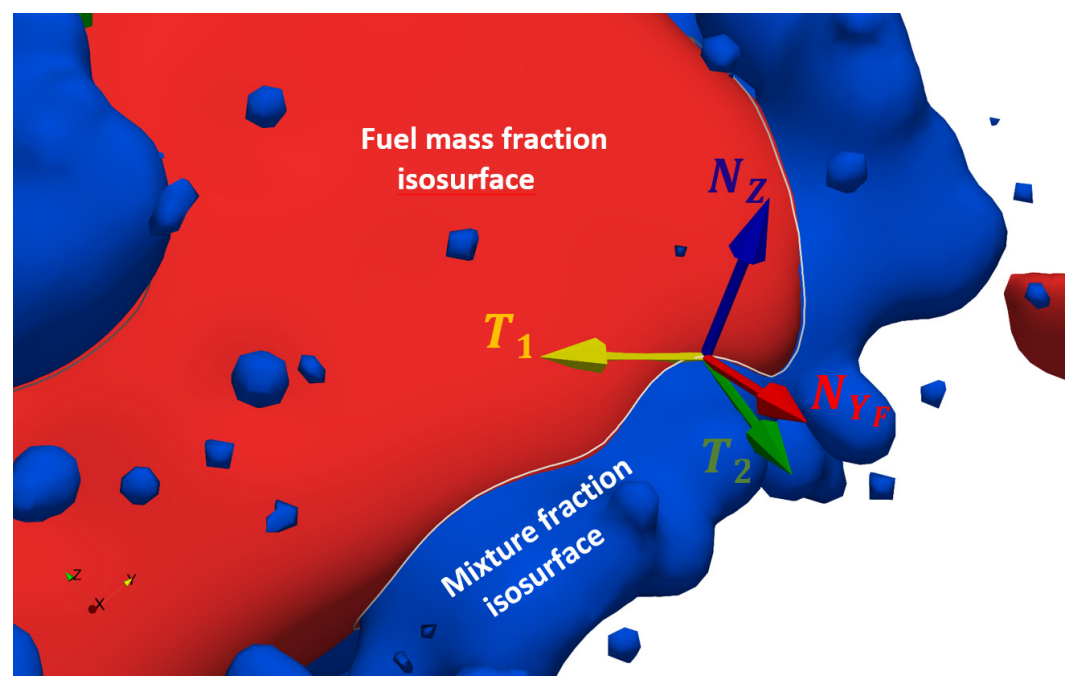

FIG. 1. A schematic diagram of the intersection between the fuel mass fraction (red) isosurface and stoichiometric mixture fraction (blue) isosurfaces and the coordinate system associated with the edge flame.

The unit vector $\boldsymbol{N}_{\boldsymbol{Z}}$ is normal to the mixture fraction iso-surface (pointing towards the oxidiser), while the unit normal vector on the fuel mass fraction iso-surface, $\boldsymbol{N}_{\boldsymbol{Y}_{\boldsymbol{F}}}$ points towards the reactants. Using these two vectors, another vector $\boldsymbol{T}_{\mathbf{1}}$ is also defined, which is normal to both $\boldsymbol{N}_{\boldsymbol{Z}}$ and $\boldsymbol{N}_{\boldsymbol{Y}_{\boldsymbol{F}}}$. Lastly, the vector $\boldsymbol{T}_{\mathbf{2}}$ is defined, which is tangential to the mixture fraction iso-surface, and normal to $\boldsymbol{T}_{\mathbf{1}}$. The expressions for the aforementioned 
vectors are given as 2425 :

$$
\begin{aligned}
\mathbf{N}_{\mathbf{Z}} & =-\frac{\nabla \xi}{|\nabla \xi|} \\
\mathbf{N}_{\mathbf{Y}_{\mathbf{F}}} & =\frac{\nabla Y_{F}}{\left|\nabla Y_{F}\right|} \\
\mathbf{T}_{\mathbf{1}} & =\frac{\mathbf{N}_{\mathbf{Y}_{\mathbf{F}}} \times \mathbf{N}_{\mathbf{Z}}}{\left|\mathbf{N}_{\mathbf{Y}_{\mathbf{F}}} \times \mathbf{N}_{\mathbf{Z}}\right|} \\
\mathbf{T}_{\mathbf{2}} & =\frac{\mathbf{N}_{\mathbf{Z}} \times\left(\mathbf{N}_{\mathbf{Y}_{\mathbf{F}}} \times \mathbf{N}_{\mathbf{Z}}\right)}{\left|\mathbf{N}_{\mathbf{Y}_{\mathbf{F}}} \times \mathbf{N}_{\mathbf{Z}}\right|}=\mathbf{N}_{\mathbf{Z}} \times \mathbf{T}_{\mathbf{1}}
\end{aligned}
$$

As outlined by Eq. 18,21 and Fig, 1, the coordinate system is attached to the edge flame, and thus only the motion of the iso-surfaces relative to the flow is considered. This relative motion arises due to the displacement of the mixture fraction and the fuel mass fraction iso-surfaces, and thus their relative velocities need to be defined. The relative velocity of the mixture fraction iso-surface is defined as $S_{Z} N_{Z}$, whilst the fuel mass fraction iso-surface moves with a velocity $S_{d} N_{\boldsymbol{Y}_{\boldsymbol{F}}}$. The displacement speeds are defined as ${ }^{1+512425}$ :

$$
\begin{aligned}
S_{d} & =-\frac{1}{\rho\left|\nabla Y_{F}\right|}\left(\dot{\omega}_{F}+M_{Y_{F}}\left(1-Y_{F}\right)+\frac{\partial}{\partial x_{i}}\left(\rho D \frac{\partial Y_{F}}{\partial x_{i}}\right)\right) \\
S_{Z} & =\frac{1}{\rho|\nabla \xi|}\left(\dot{A}_{\Gamma_{Y_{F}}}+\frac{\partial}{\partial x_{i}}\left(\rho D \frac{\partial \xi}{\partial x_{i}}\right)\right)
\end{aligned}
$$

which follow definitions in existing literature ${ }^{3 / 4144}$. However due to the presence of droplets, the additional terms $M_{Y_{F}}\left(1-Y_{F}\right)$ and $\dot{A}_{\Gamma_{Y_{F}}}$ are added. Here, $\dot{\omega}_{F}$ is the fuel reaction rate, $D$ is the molecular diffusivity, $M_{Y_{F}}$ is the evaporation rate of the droplets that arises due to mass conservation from the transport equation of the scalar quantity $Y_{F}$. The term $\dot{A}_{\Gamma_{Y_{F}}}=\left[M_{Y_{F}}\left(1-Y_{F}+M_{Y_{F}} Y_{O} / s\right)\right] /\left(Y_{F_{\infty}}+Y_{O_{\infty}} / s\right)$ is a source term that accounts for the change of mixture fraction due to droplet evaporation. The overall velocity of the edge point on the stoichiometric mixture fraction (i.e. $\xi=\xi_{s t}$ ) isosurface is defined as $\boldsymbol{V}_{\boldsymbol{e}}$, and is decomposed into orthonormal coordinates $\boldsymbol{N}_{\boldsymbol{Z}}$ and $\boldsymbol{T}_{\mathbf{2}}$ as ${ }^{2425}$ :

$$
\mathbf{V}_{\mathbf{e}}=S_{Z} \mathbf{N}_{\mathbf{Z}}+S_{e} \mathbf{T}_{\mathbf{2}}
$$

where $S_{e}=\boldsymbol{V}_{\boldsymbol{e}} \cdot \boldsymbol{T}_{\mathbf{2}}$ is the projection of $\boldsymbol{V}_{\boldsymbol{e}}$ into the plane tangential to the mixture fraction iso-surface. By taking the dot product of Eq. 24 with $\boldsymbol{N}_{\boldsymbol{Y}_{\boldsymbol{F}}}$, the edge flame speed $S_{e}$ on the stoichiometric mixture fraction (i.e. $\xi=\xi_{s t}$ ) isosurface is obtained as ${ }^{24 \mid 25}$ :

$$
S_{e}=\frac{S_{d}-k S_{Z}}{\sqrt{1-k^{2}}}
$$


where $k=N_{\boldsymbol{Y}_{\boldsymbol{F}}} \cdot \boldsymbol{N}_{\boldsymbol{Z}}$ is the inner product of the normal vectors. To investigate the physical mechanisms which affect the edge flame propagation, it is worthwhile to define different components of $S_{e}=\left(S_{d}^{*}+S_{Z}^{*}\right), S_{d}=\left(S_{d_{\text {reac }}}+S_{\text {devap }}+S_{d_{\text {diff }}}\right)$ and $S_{Z}=\left(S_{Z_{\text {evap }}}+S_{Z_{\text {diff }}}\right)$ :

$$
\begin{gathered}
S_{d}^{*}=\frac{S_{d}}{\sqrt{1-k^{2}}}, \\
S_{Z}^{*}=\frac{-k S_{Z}}{\sqrt{1-k^{2}}} \\
S_{d_{\text {reac }}}=-\frac{\dot{\omega}_{F}}{\rho\left|\nabla Y_{F}\right|}, S_{d_{\text {evap }}}=-\frac{M_{Y_{F}}\left(1-Y_{F}\right)}{\rho\left|\nabla Y_{F}\right|}, S_{d_{\text {diff }}}=-\frac{\nabla \cdot\left(\rho D \nabla Y_{F}\right)}{\rho\left|\nabla Y_{F}\right|} \\
S_{Z_{\text {evap }}}=\frac{\dot{A}_{\Gamma_{Y_{F}}}}{\rho|\nabla \xi|}, S_{Z_{\text {diff }}}=\frac{\nabla \cdot(\rho D \nabla \xi)}{\rho|\nabla \xi|}
\end{gathered}
$$

where the subscripts reac, evap and diff represent reaction, evaporation and diffusion components respectively. The diffusion components of both $S_{d}$ and $S_{Z}$ can be further decomposed into normal and tangential components (i.e. $S_{d_{d i f f}}=\left(S_{d_{n o r m}}+S_{d_{t a n g}}\right)$ and $\left.S_{Z_{\text {diff }}}=\left(S_{Z_{\text {norm }}}+S_{Z_{\text {tang }}}\right)\right)$ as shown below :

$$
\begin{aligned}
& S_{d_{\text {norm }}}=-\frac{1}{\rho\left|\nabla Y_{F}\right|^{2}}\left(\frac{\partial Y_{F}}{\partial x_{j}} \frac{\partial\left(\rho D\left|\nabla Y_{F}\right|\right)}{\partial x_{j}}\right), S_{Z_{\text {norm }}}=\frac{1}{\rho|\nabla \xi|^{2}}\left(\frac{\partial \xi}{\partial x_{j}} \frac{\partial(\rho D|\nabla \xi|)}{\partial x_{j}}\right) \\
& S_{d_{\text {tang }}}=-2 D \kappa_{Y, m}, S_{Z_{\text {tang }}}=-2 D \kappa_{Z, m}
\end{aligned}
$$

where subscripts norm and tang indicate normal and tangential components, respectively and $\kappa_{Y, m}$ and $\kappa_{Z, m}$ are defined as ${ }^{115}$ :

$$
\begin{aligned}
& \kappa_{Y, m}=0.5 \nabla \cdot \mathbf{N}_{\mathbf{Y}_{\mathbf{F}}}, \\
& \kappa_{Z, m}=0.5 \nabla \cdot \mathbf{N}_{\mathbf{Z}}
\end{aligned}
$$

Equations 32 and 33 provide the expressions for the curvatures of the fuel mass fraction and mixture isosurfaces, respectively. In addition to curvature, the edge flame speed behaviour is influenced by the tangential strain rates acting on the mixture fraction and on the fuel mass fraction isosurfaces (i.e. $a_{t, Y}$ and $a_{t, Z}$ respectively). The tangential strain rates $a_{t, Y}$ and $a_{t, Z}$ are defined as ${ }^{115}$ :

$$
\begin{aligned}
& a_{t, Y}=\left(\delta_{i j}-N_{Y_{F}, i} N_{Y_{F}, j}\right) \frac{\partial u_{i}}{\partial x_{j}}, \\
& a_{t, Z}=\left(\delta_{i j}-N_{Z, i} N_{Z, j}\right) \frac{\partial u_{i}}{\partial x_{j}}
\end{aligned}
$$

The effects of curvature and tangential strain rate on the edge flame speed $S_{e}$ will be discussed in detail in Section 4 of this paper. 


\section{NUMERICAL IMPLEMENTATION}

The simulations have been carried out using the three-dimensional compressible DNS code SENGA+1 ${ }^{1 / 5}$ in a domain of size $51 \delta_{z} \times 51 \delta_{z} \times 51 \delta_{z}$. The domain is discretized by a uniform Cartesian grid of $264 \times 264 \times 264$ cells, which ensures 10 grid points across the thermal flame thickness of the stoichiometric mixture $\delta_{s t}=\left[T_{a d_{\phi_{g}=1}}-T_{0}\right] / \max \left(|\nabla \hat{T}|_{L}\right)$. It also ensures $\eta_{k}>\Delta x$, where $\eta_{k}$ is the Kolmogorov length scale and $\Delta x$ is the DNS grid spacing. For these simulations, all the boundaries of the domain are considered to be partially non-reflecting and are specified using the Navier-Stokes Characteristic Boundary Conditions (NSCBC) technique $e^{36}$. The spatial differentiation and time advancements have been carried out using high-order finite difference $\left(10^{t h}\right.$-order central difference for the internal grid points and the order of differentiation gradually decreases to $2^{\text {nd }}$-order one-sided scheme at the boundaries) and $3^{r d}$-order low-storage explicit Runge-Kutta schemes ${ }^{37}$, respectively. The flame-turbulence interaction takes place under decaying isotropic homogeneous turbulence. A well-known pseudo-spectral method ${ }^{38}$ is used to initialise the turbulent velocity fluctuations by an incompressible, homogeneous isotropic field with prescribed values of root-mean-square (rms) values $u^{\prime}$ and integral length scale $L_{11}$.

The initial mixture consists of n-heptane liquid droplets uniformly dispersed in air and the overall equivalence ratio $\phi_{o v}$ is determined by the number of droplets initially present in the domain. Three different droplet diameters $a_{d}$ are considered $\left(a_{d} / \delta_{s t}=0.02,0.03,0.04\right)$, across three different global equivalence ratios $\left(\phi_{o v}=0.8,1.0,1.2\right)$. The normalised initial droplet number densities $\rho_{N}$ for these cases are given by : $1.79 \leq\left(\rho_{N}\right)^{1 / 3} \delta_{s t} \leq 3.81$ in the unburned gas, and the percentage of liquid volume remains well below $0.01 \%$. The corresponding mass loading can be obtained from the overall equivalence ratio, as the fuel is supplied in the form of liquid droplets. The liquid to gas ratio by mass remains smaller than 7.5\% for the cases considered here. All three droplet diameters and global equivalence ratios are investigated across three different initial turbulence intensities $\left(u^{\prime} / S_{L_{\phi_{g}=1}}=0.0,4.0,8.0\right)$. For all turbulent cases, the initial value of $L_{11} / \delta_{z}$ is taken to be 5.31 (i.e. $L_{11} / \delta_{z}=5.31$ ), which ensures about 10 large scale eddies on each side of the domain.

For ease of reference and brevity, each case will be referred to in the following manner : U00, U04, U08 refer to initial turbulence intensities of $u^{\prime} / S_{L_{\phi_{g}=1}}=0.0,4.0,8.0$ respectively; D02, D03, D04 refer to initial droplet diameters $a_{d} / \delta_{s t}=0.02,0.03,0.04$, respectively, and 
F08, F10, F12 refer to $\phi_{\text {ov }}=0.8,1.0,1.2$ respectively.

The unburned gas temperature $T_{0}$ is taken to be $300 \mathrm{~K}$, which yields a heat release parameter of $\tau=6.54$, at atmospheric pressure. Care was taken to ensure that the droplet diameter remains smaller than the Kolmogorov length scale for all cases investigated, and the ratio of initial droplet diameter to the Kolmogorov length scales is $a_{d} / \eta_{k}=0.15,0.22,0.29$ for $a_{d} / \delta_{s t}=0.02,0.03,0.04$, respectively, for the highest initial turbulence intensity investigated here. The ratios $a_{d} / \eta_{k}$ as well as $a_{d} / \Delta x$ remain comparable to several previous analyses by other authors $5 \sqrt[51 / 39]{44}$, and thus the assumption of sub-grid point sources (similar to Refs. $30 \mid 32[39 \mid 49]$ ) is not expected to significantly affect the ignition phenomenon and subsequent flame-droplet interaction. The Stokes number, defined as $S t=\left(\tau_{p} \sqrt{k_{e}}\right) / L_{11}=$ $\left(\rho_{d} a_{d}^{2} \sqrt{k_{e}}\right) /\left(18 C_{u} \mu L_{11}\right)$ (where $\tau_{p}=\left(\rho_{d} a_{d}^{2}\right) /\left(18 C_{u} \mu\right)$ is the particle time scale and $L_{11} / \sqrt{k_{e}}$ is the turbulent time scale with $k_{e}$ being the turbulent kinetic energy evaluated over the whole volume) remains smaller than 0.36 for the largest droplet in the highest turbulence intensity case. An alternative Stokes number $S t^{\prime}=\left(\tau_{p} S_{L_{\phi_{g}=1}}^{2}\right) / \alpha_{T_{0}}=\left(\rho_{d} a_{d}^{2} S_{L_{\phi_{g}=1}}^{2}\right) /\left(18 C_{u} \mu \alpha_{T_{0}}\right)$ based on the chemical time scale $\alpha_{T_{0}} / S_{L_{\phi_{g}=1}}^{2}$ remains smaller than 0.21 for the largest droplets considered in this analysis. The mean normalised inter-droplet distance $s_{i d} / \eta_{k}$ initially varies between 2.26 and 2.85 for the cases considered here. Due to the high volatility of n-heptane, the size of the droplets decreases significantly (up to $50 \%$ from their initial diameter in the unburned gas) by the time they reach the reaction zone. Thus, the droplets, which interact with the flame, are much smaller in reality than the initial size of the droplets leading to the conclusion that the assumption of sub-grid point sources is not expected to affect the statistics of flame-droplet interaction analysed in this paper.

The edge flame statistics have been extracted at $t=8.0 t_{s p}$ which is well after the end of the energy deposition, and amounts to $1.6 t_{f}$ which is about $1.5 L_{11} / \sqrt{k_{e}}\left(3.0 L_{11} / \sqrt{k_{e}}\right)$ for the initial $u^{\prime} / S_{L_{\phi_{g}=1}}=4.0$ (8.0) cases. Thus, the energy deposited by the ignitor is not expected to interfere with the edge flame statistics. By that time, $u^{\prime}$ evaluated over the whole domain had decayed by $25 \%$ and $38 \%$ in comparison to its initial value for the U04 and U08 cases, respectively, while the turbulent kinetic energy and its dissipation rate were not changing rapidly with time at $t=8.0 t_{s p}$ when the edge flame statistics were extracted for all cases considered here. This simulation time remains comparable to several previous DNS analyses on localised forced ignition $1 \frac{16[32 / 45 / 46 / 50 \mid}{52}$. 


\section{RESULTS \& DISCUSSION}

In the present paper, the temporal evolution of maximum temperature $T_{\max }$ is not shown for the sake of brevity but the corresponding distribution looks qualitatively similar to that presented in several previous analyses 1 6150. In all cases, the thermal runaway takes place within the energy deposition period and the value of $T_{\max }$ peaks above unity (i.e. a value greater than the adiabatic flame temperature of the stoichiometric mixture) at the end of energy deposition for the considered values of the spark parameters (i.e. $a_{s p}, b_{s p}$ and $R_{s p}$ ). Once the ignitor is switched off, the maximum temperature $T_{\max }$ decreases with time and eventually relaxes to a value of unity during the early stages of the self-sustained combustion process, which is attained in all cases considered here.

\section{A. Flame morphology and composition of gaseous phase mixture}

The three-dimensional views of the $c=0.5$ isosurface at the time instant $t=8.0 t_{s p}$ are exemplarily shown in Fig. 2 for all cases with $\phi_{o v}=1.0$ in order to demonstrate the effects of droplet diameter $a_{d}$ on the flame morphology for different turbulence intensities. The effects of $\phi_{o v}$ are demonstrated in Fig. 3 where the three-dimensional views of the $c=0.5$ isosurfaces at the time instant $t=8.0 t_{s p}$ are shown for all the cases with an initial normalised droplet diameter of $a_{d} / \delta_{s t}=0.03$. It can be discerned from Figs. 2 , 3 that the droplets induce flame wrinkling, which can be seen in the form of dimples on the $c$-isosurface for the laminar and initial $u^{\prime} / S_{L_{\phi_{g}=1}}=4.0$ cases. This droplet-induced flame wrinkling becomes increasingly eclipsed by the wrinkling due to turbulent fluid motion with increasing turbulence intensity. As turbulence intensity increases, fluid motion wrinkles and deforms the flame significantly from its original spherical shape (as observed for the laminar cases). Moreover, the dropletinduced flame wrinkling strengthens with increasing $a_{d}$ and $\phi_{o v}$ for both laminar and low turbulence intensity cases. The observations related to droplet-induced flame wrinkling are consistent with previous experimental $\sqrt[53 \mid 54]{ }$ and numerical ${ }^{47 / 48}$ findings.

In Figs. 2-3, the $c=0.5$ isosurfaces are coloured by the local values of the modified flame index, which is defined as 5

$$
\psi=\frac{1}{2} \frac{\xi-\xi_{s t}}{\left|\xi-\xi_{s t}\right|}\left(1+\frac{\nabla Y_{F} \cdot \nabla Y_{O}}{\left|\nabla Y_{F}\right| \cdot\left|\nabla Y_{O}\right|}\right)
$$




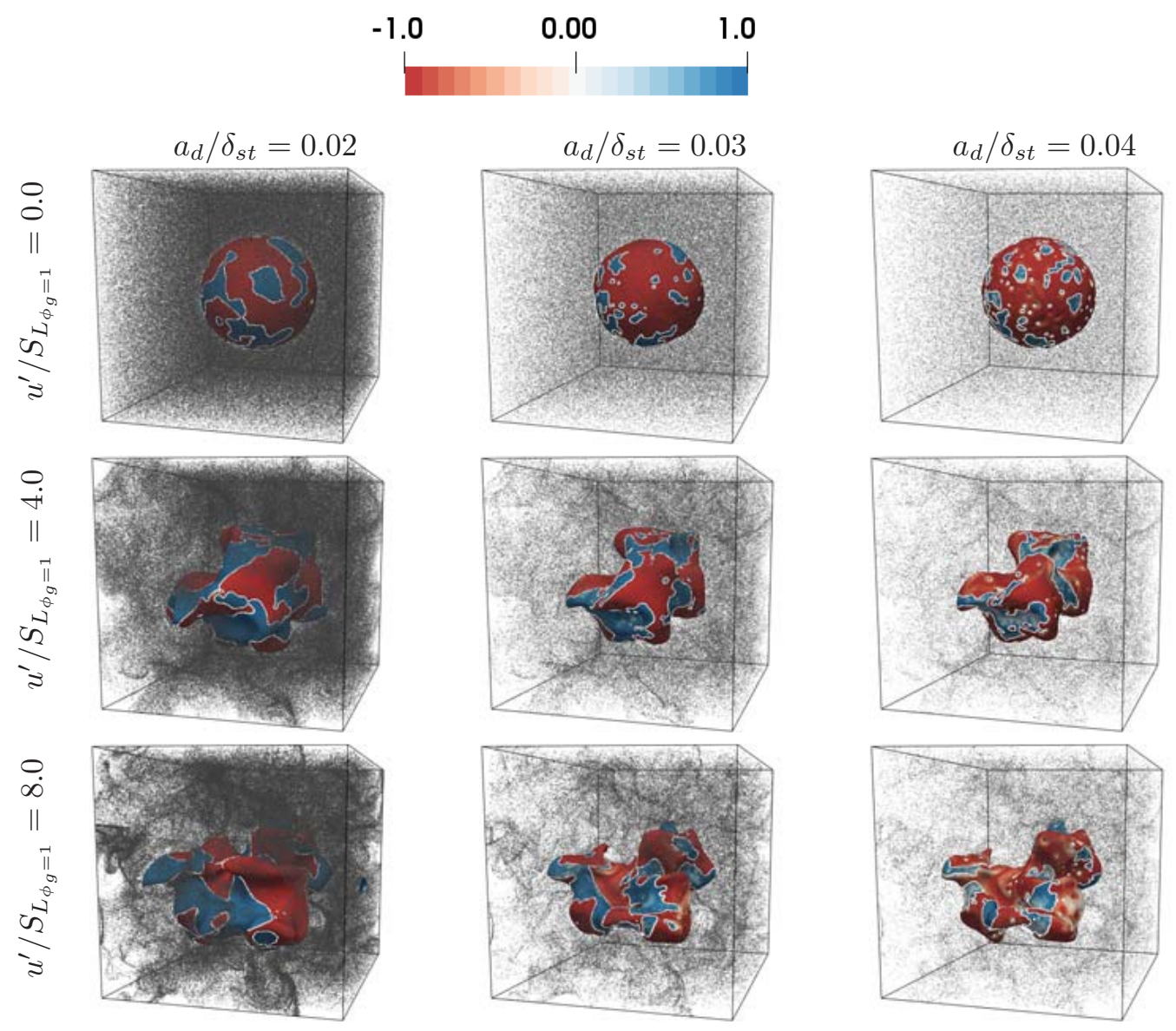

FIG. 2. Instantaneous views of $c=0.5$ isosurface coloured by local values of flame index $\psi$ at $t=8.0 t_{s p}$ for $\phi_{o v}=1.0$ cases with laminar, initial $u^{\prime} / S_{L_{\phi_{g}=1}}=4.0$ and initial $u^{\prime} / S_{L_{\phi_{g}=1}}=8.0$ (from top to bottom) for initial normalised diameters of $a_{d} / \delta_{s t}=0.02,0.03$ and 0.04 (from left to right). The stoichiometric mixture fraction is shown using the solid white line.

According to the definition, $\psi=-1.0$ and $\psi=1.0$ indicate fuel-lean and fuel-rich premixed combustion respectively, whereas $\psi=0$ is the indicative of non-premixed combustion. It is evident from Figs. 2 3 3 that rich-premixed and lean-premixed flames co-exist on the chosen $c$ isosurface and a diffusion (i.e. non-premixed) mode of burning can be seen between the premixed flames. Moreover, this diffusion mode of burning is obtained where the mixture fraction in the gaseous phase attains the stoichiometric value (i.e. $\xi=\xi_{s t}$ ). Thus in Figs. 2,3, the locations where a diffusion mode of burning is observed, overlap with the line indicating the stoichiometric mixture fraction value. This behaviour is indicative of the presence of 
multiple edge flame structures. It can further be seen from Figs. 2 3 that turbulence intensity, droplet size and overall equivalence ratio have significant influences on the occurrence and spread of edge flames.

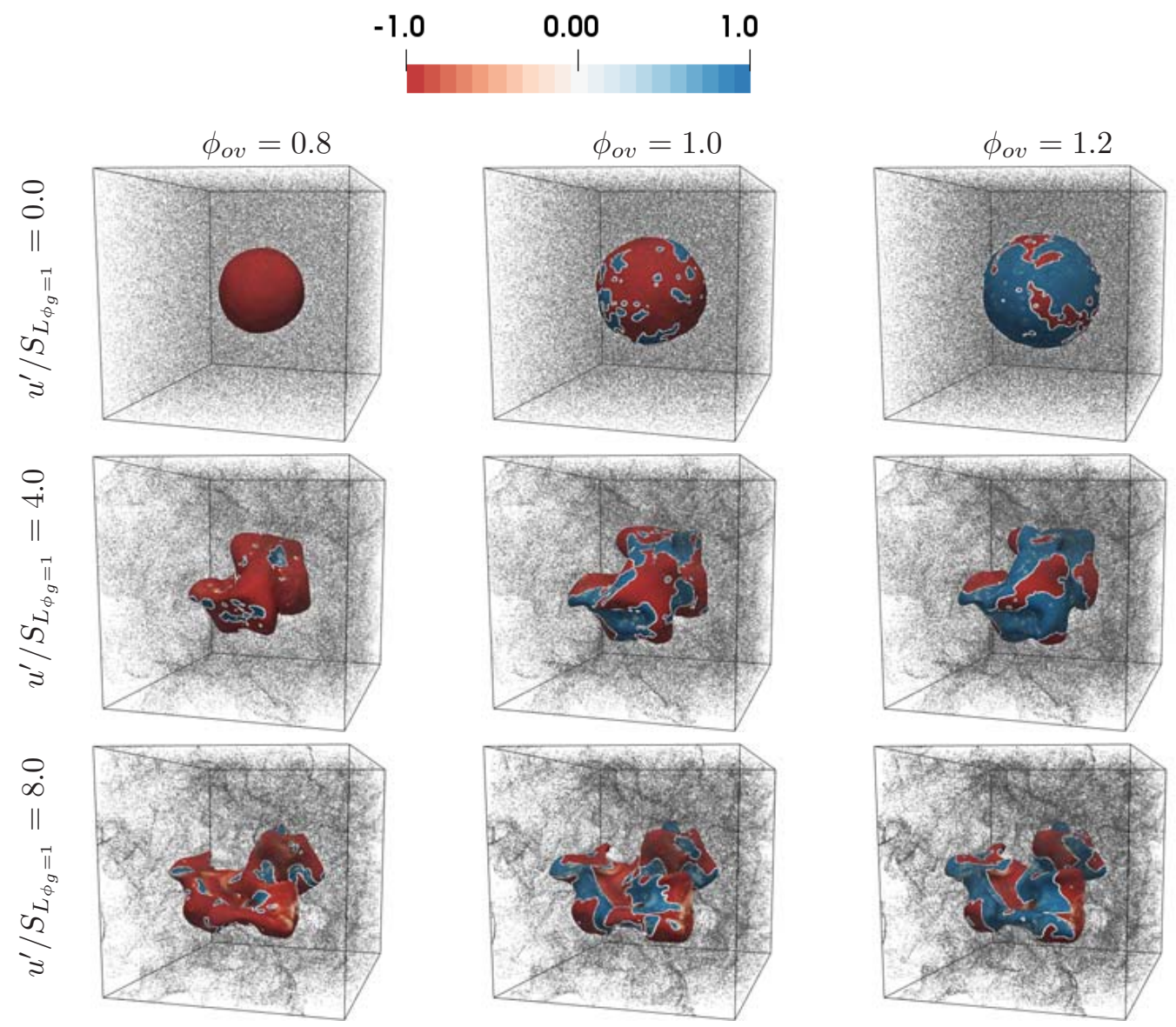

FIG. 3. Instantaneous views of $c=0.5$ isosurface coloured by local values of flame index $\psi$ at $t=8.0 t_{s p}$ for $a_{d} / \delta_{s t}=0.03$ cases with laminar, initial $u^{\prime} / S_{L_{\phi_{g}=1}}=4.0$ and initial $u^{\prime} / S_{L_{\phi_{g}=1}}=8.0$ (from top to bottom) for overall equivalence ratios $\phi_{o v}=0.8,1.0$ and 1.2 (from left to right). The stoichiometric mixture fraction is shown using the solid white line.

The edge flame development with the variation in $c$ is exemplarily shown in Fig. 4 for the U04D03F08 case where the isosurfaces of $c$ are coloured by the local values of flame index $\psi$ and fuel mass fraction isosurface curvature $\kappa_{m, Y}$. It can be seen from Fig. 4 that the edge flame is obtained for small values of $c$ close to the dimples on the reaction progress variable isosurface created by the evaporation of droplets and these regions are predominantly asso- 
ciated with large negative values of $\kappa_{m, Y}$. However, the edge flame spreads and spans over larger patches for higher values of $c$ due to greater extent of mixing and also because of the greater availability of the gaseous fuel vapour. Towards the burned gas side, the edge flame does not remain confined only to the dimples created by the evaporation of droplets and the edge flame locations exhibit predominantly positive values of $\kappa_{m, Y}$.
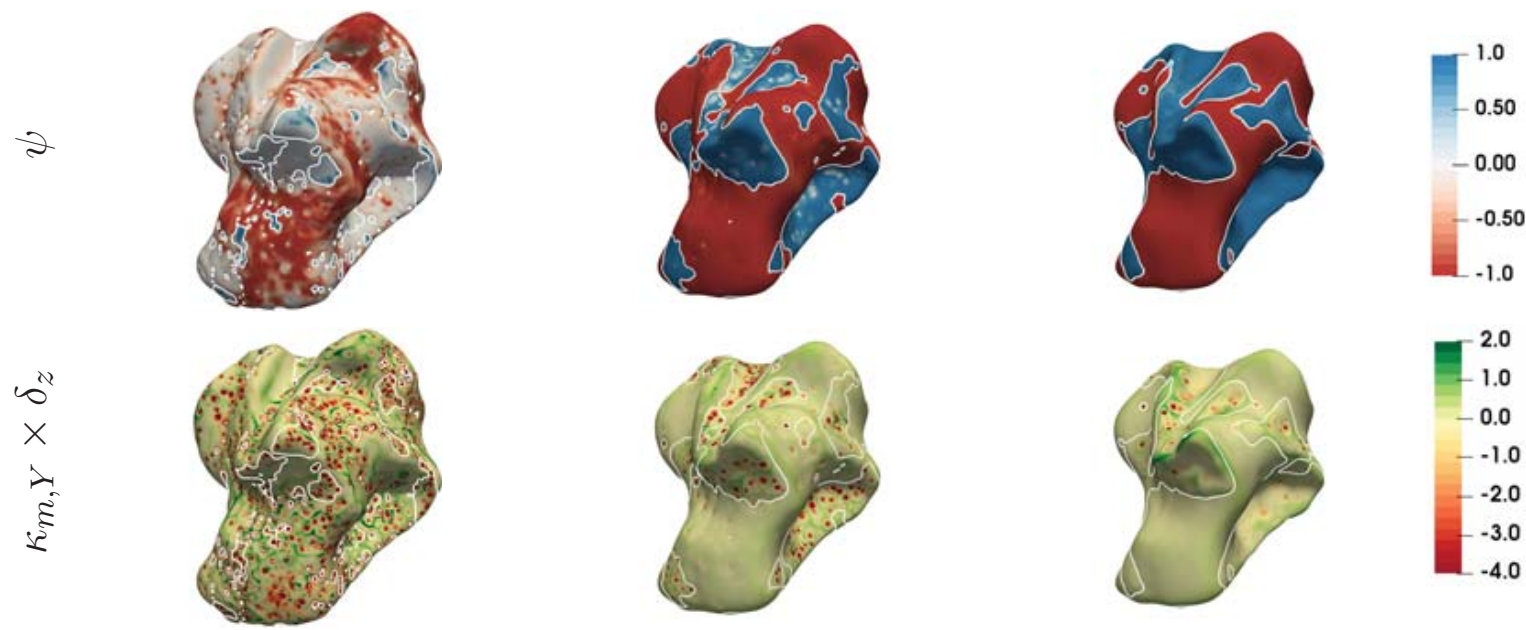

FIG. 4. Instantaneous views of $c=0.15, c=0.50$ and $c=0.85$ (left to right) isosurfaces coloured by local values of flame index $\psi\left(1^{\text {st }}\right.$ row $)$ and normalised curvature of the fuel mass fraction isosurface $\kappa_{m, Y} \times \delta_{z}\left(2^{\text {nd }}\right.$ row $)$ at $t=8.0 t_{s p}$ for the $\mathrm{U} 04 \mathrm{D} 03 \mathrm{~F} 10$ case. The stoichiometric mixture fraction is shown using the solid white line.

The above discussion suggests that the mixture composition in the gaseous phase plays a key role in the possibility of having an edge flame and the combustion behaviour subsequent to successful thermal runaway. In order to analyse the effects of $\phi_{o v}, a_{d} / \delta_{s t}$ and $u^{\prime} / S_{L_{\phi_{g}=1}}$ on the gaseous mixture composition, the normalised Probability Distribution Functions (PDFs) of equivalence ratio in the gaseous phase $\phi_{g}$ are shown in Fig. 5. In Fig. 5, the PDFs have been normalised by their respective maximum values (i.e. $P D F_{\max }$ ) because of the significant differences in the maximum PDF values, $P D F_{\text {max }}$, and thus this normalisation helps to compare the distributions of $\phi_{g}$ in the same scale. It can be substantiated from Fig. 5 that the probability of finding $\phi_{g}<\phi_{o v}$ is predominant in all cases considered here. However, there are finite probabilities of obtaining $\phi_{g}>\phi_{o v}$ for all cases but this tendency is more prominent for larger droplet sizes and turbulence intensities. It can be seen from the PDFs of $\phi_{g}$ in Fig. 5 that the probability of finding $\phi_{g}=1.0$ is either zero or negligible 
for laminar cases and the initial $u^{\prime} / S_{L_{\phi_{g}=1}}=4.0$ case with initial $a_{d} / \delta_{s} t=0.02$ for $\phi_{o v}=0.8$ (e.g. U00D02F08, U00D03F08, U00D04F08 and U04D02F08). However, a combination of the dispersion process by turbulent fluid motion and slow evaporation rate for large droplets produces local occurrences $\phi_{g}=1.0$ even for $\phi_{o v}=0.8$ (e.g. U04D03F08, U08D02F08, U08D03F08 and U08D04F08) at high values of $u^{\prime} / S_{L_{\phi_{g}=1}}$. The occurrence of edge flame is less prominent or absent in the laminar cases for $\phi_{o v}=0.8$ (e.g. U00D02F08, U00D03F08 and U00D04F08) because of either rarity or absence of finding a stoichiometric mixture within the flame front. Thus, the edge flame statistics have not been analysed for these cases. Due to the lean overall equivalence ratio $\left(\phi_{o v}=0.8\right)$ for these cases, which corresponds to a smaller number of droplets being initially present, the energy deposited is more than sufficient to fully evaporate all the droplets initially present within the energy deposition region. The diffusive transport is relatively weak under laminar conditions, and thus all the deposited energy contributes towards evaporating the droplets in the vicinity of the ignitor, and initiating combustion of the evaporated fuel vapour. As a result, the flame front encounters a predominantly gaseous mixture under fuel-lean conditions, which implies that the local mixture fractions encountered across the flame front remain smaller than the stoichiometric mixture fraction value. Moreover, the rapid evaporation rate of small droplets leads to a more homogeneous fuel-air mixture upon mixing the freshly evaporated fuel vapour with surrounding air in comparison to the cases with larger droplets ${ }^{47}$. This gives rise to the predominance of fuel-lean stratified gaseous mixtures in the $\phi_{o v}=0.8$ cases and thus the occurrence of edge flame is unlikely in these cases. However, dispersion of evaporated fuel vapour from the droplets under highly turbulent (e.g. $u^{\prime} / S_{L_{\phi_{g}=1}}=8.0$ ) cases can lead to local occurrences of gaseous equivalence ratios $\phi_{g}$ which are fuel-richer than suggested by the overall equivalence ratio $\phi_{o v}$ (i.e. $\left.\phi_{g}>\phi_{o v}\right)^{48}$. This leads to finite probabilities of finding $\phi_{g}=1.0$ (i.e. $\xi=\xi_{s t}$ ) and edge flames in the $\phi_{o v}=0.8$ cases with an initial turbulence intensity of $u^{\prime} / S_{L_{\phi_{g}=1}}=8.0$.

\section{B. Marginal PDFs of edge flame speed and its components}

The PDFs of normalised edge flame speed $S_{e} / S_{L_{\phi_{g}=1}}$ for all cases considered here except for the U00D02F08, U00D03F08 and U00D04F08 cases (reasons are provided earlier) are shown in Fig. 6. It has been analytically derived by Ruetsch et al ${ }^{11}$ that the laminar 

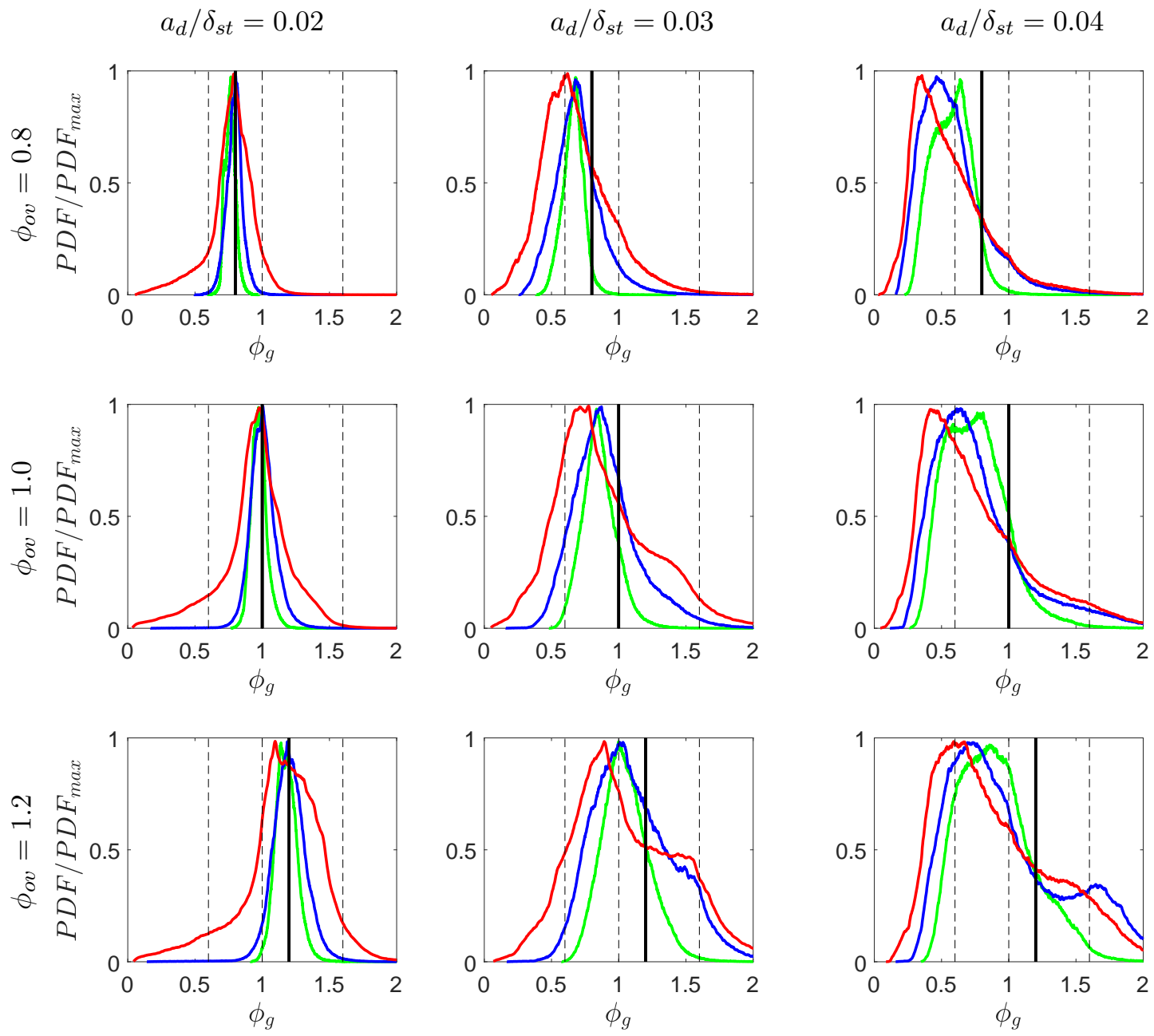

FIG. 5. PDFs of equivalence ratio in the gaseous phase $\phi_{g}$ normalised by their respective maximum values (i.e. $P D F / P D F_{\max }$ ) in the region given by $0.1 \leq c \leq 0.9$ for initial $a_{d} / \delta_{s t}=0.02,0.03$ and 0.04 (left to right) and overall equivalence ratios $\phi_{o v}=0.8,1.0$ and 1.2 (top to bottom), in the case of laminar (green), initial $u^{\prime} / S_{L_{\phi_{g}=1}}=4.0$ (blue) and initial $u^{\prime} / S_{L_{\phi_{g}=1}}=8.0$ (red) conditions. The vertical dashed black lines indicate flammability limits and stoichiometry, with the bold solid black line, indicating initial $\phi_{o v}$ for each set of cases respectively.

edge flame speed is given by $S_{e}^{0}=S_{L_{\phi_{g}=1}} \sqrt{\rho_{0} / \rho_{b}}$ where $\rho_{0}$ is the unburned gas density and $\rho_{b}$ represents the burned gas density of the stoichiometric mixture, which leads to $S_{e}^{0} / S_{L_{\phi_{g}=1}}=\sqrt{\rho_{0} / \rho_{b}} \approx \sqrt{\tau+1}$. For the present study $S_{e}^{0} / S_{L_{\phi_{g}=1}} \approx 2.73$, and is indicated in Fig. 6 by the vertical broken black line. As can be observed from Fig. 6, the peaks of the PDFs of $S_{e}$ for the laminar cases (green lines) are in good agreement with the theoretical 
estimate of $S_{e}^{0} / S_{L_{\phi_{g}=1}}$. Even though the most probable value of $S_{e} / S_{L_{\phi_{g}=1}}$ remains close to $\sqrt{\rho_{0} / \rho_{b}} \approx 2.73$ for turbulent cases, the most probable value of $S_{e}$ decreases from the laminar flame estimate $S_{L_{\phi_{g}=1}} \sqrt{\rho_{0} / \rho_{b}} \approx 2.73$ for turbulent cases and this deviation increases with increasing turbulence intensity. The peak value of the PDF of $S_{e}$ decreases and the width of the PDF increases with increasing $u^{\prime} / S_{L_{\phi_{g}=1}}$ as the scatter of $S_{e}$ around the mean value increases due to local variations in strain rate, scalar gradient and curvature, which are known to affect the edge flame speed in gaseous single-phase partially-premixed flames 1317117 19242025. It can further be seen from Fig. 6 that both the initial droplet diameter $a_{d}$ and the overall equivalence ratio $\phi_{o v}$ do not appreciably affect the qualitative distributions of $S_{e} / S_{L_{\phi_{g}=1}}$ for both laminar and turbulent flames. However, the droplet size and overall equivalence ratio have already been shown to influence the gaseous phase composition, which along with droplet-induced flame deformation (and thereby affect the flame curvature distribution) and proportions of different $c$ values within the sample corresponding to $0.1 \leq c \leq 0.9$ gives rise to a range of different values of $S_{e} / S_{L_{\phi_{g}=1}}$ in laminar cases. An increase in $a_{d}$ for a given number density reduces the overall evaporation rate and thus the evaporated fuel in the gaseous phase mixes imperfectly with the surrounding air, which in turn gives a greater likelihood of non-premixed mode of combustion occurring. Thus, the typical edge flame structure becomes increasingly likely for large droplets and this tendency increases further with increasing $\phi_{o v}$ because of the larger number density of droplets. This, in turn, gives rise to a sharper peak of the PDFs of $S_{e} / S_{L_{\phi_{g}=1}}$ at $S_{e} / S_{L_{\phi_{g}=1}}=\sqrt{\rho_{0} / \rho_{b}} \approx 2.73$ for larger droplet diameters and this trend strengthens further with increasing $\phi_{o v}$.

It has already been shown in Eqs. 2527 that $S_{e}$ can be decomposed as $S_{e}=S_{d}^{*}+S_{Z}^{*}$. Thus, the PDFs of $S_{d}^{*}$ are also shown in Fig. 6 by the dotted lines. It can be seen from Fig. 6 that the PDFs of $S_{e} / S_{L_{\phi_{g}=1}}$ and $S_{d}^{*} / S_{L_{\phi_{g}=1}}$ are nearly identical implying that the contributions of $S_{Z}^{*}$ towards the $S_{e}$ PDFs are negligible. The PDFs of $S_{Z}^{*}$ are not shown separately here for the sake of conciseness but these PDFs peak at zero value confirming that the contributions of $S_{Z}$ to the mean edge flame speed $S_{e}$ are negligible for all cases considered here.

It can be seen from Fig. 6 6 that the PDFs of both $S_{e}$ and $S_{d}^{*}$ exhibit considerable probability of finding negative values which suggests that the flame locally retreats into the burned gas instead of propagating into the unburned gas mixture. Moreover, the probability of finding negative $S_{e}$ and $S_{d}^{*}$ increases with increasing turbulence intensity. However, in all cases 

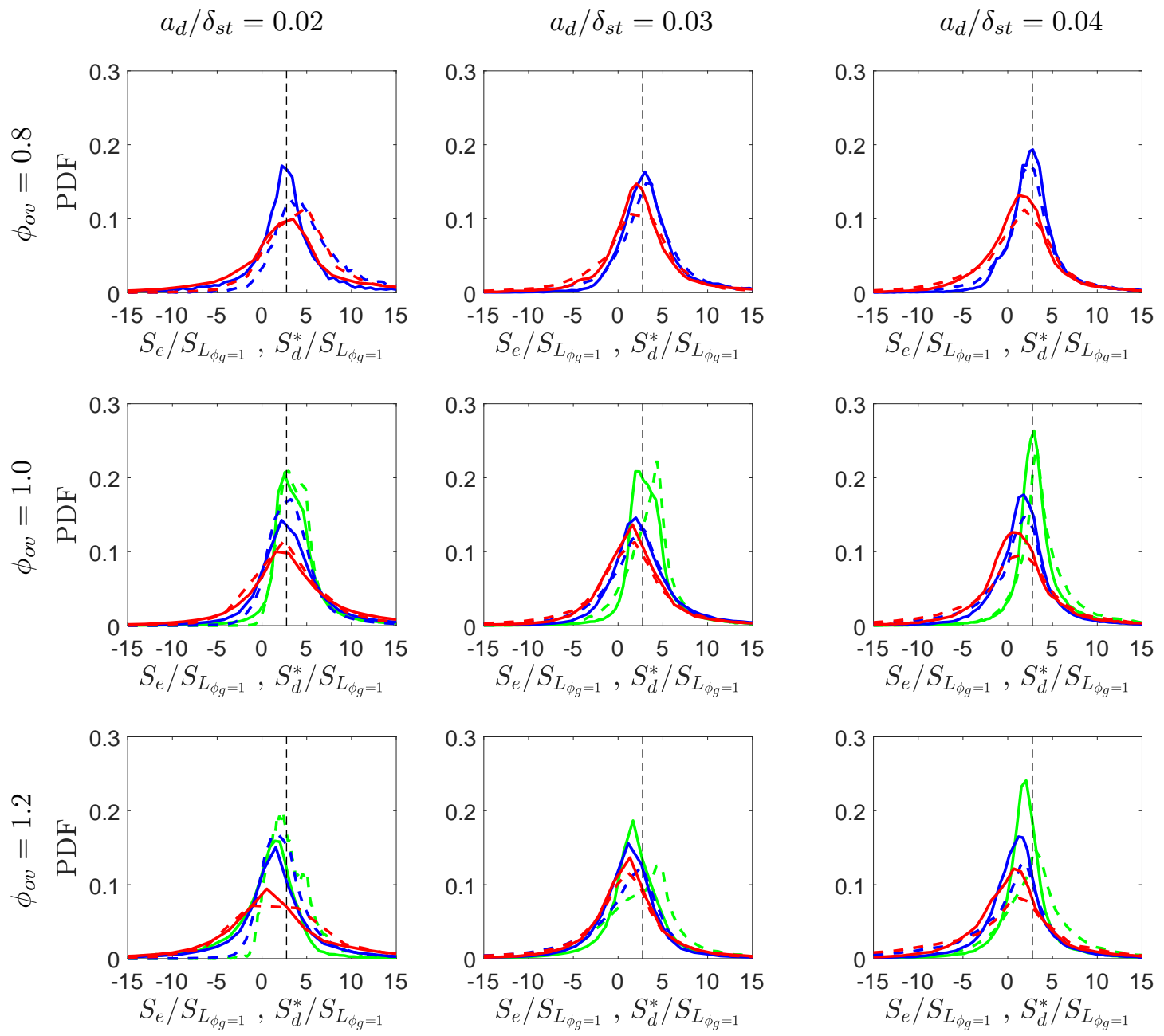

FIG. 6. PDFs of $S_{e} / S_{L_{\phi_{g}=1}}$ (solid line) and $S_{d}^{*} / S_{L_{\phi_{g}=1}}=S_{d} /\left(S_{L_{\phi_{g}=1}} \sqrt{1-k^{2}}\right)$ (broken line) based on the samples corresponding to $0.1 \leq c \leq 0.9$ on the stoichiometric mixture fraction (i.e. $\xi=\xi_{s t}$ ) isosurface for initial $a_{d} / \delta_{s t}=0.02,0.03$ and 0.04 (left to right) and overall equivalence ratios $\phi_{o v}=0.8,1.0$ and 1.2 (top to bottom), in the case of laminar (green), initial $u^{\prime} / S_{L_{\phi_{g}=1}}=4.0$ (blue) and initial $u^{\prime} / S_{L_{\phi_{g}=1}}=8.0$ (red) conditions. The broken vertical black line indicates the theoretical laminar flame value $S_{e}^{0} / S_{L_{\phi_{g}=1}}=\sqrt{1+\tau}$.

the mean values of $S_{e}$ and $S_{d}^{*}$ remain positive indicating that the flame propagates into the unburned mixture in a mean sense, which is indicative of the self-sustained flame propagation following localised forced ignition. As $S_{d}^{*}$ is the principal contributor to $S_{e}$, it is worthwhile to consider different components of displacement speed $S_{d}$ to explain the observed behaviours 
of the edge flame speed PDFs.

\section{Marginal PDFs of displacement speed of fuel mass fraction isosurface and its components}

In order to explain the statistical distributions of the different components of $S_{d} / S_{L_{\phi_{g}=1}}$ (i.e. $S_{d_{\text {reac }}} / S_{L_{\phi_{g}=1}}, S_{d_{\text {evap }}} / S_{L_{\phi_{g}=1}}$, and $S_{d_{d i f f}} / S_{L_{\phi_{g}=1}}$ ), it is worthwhile to consider their mean variations conditional on $c$, which are exemplarily shown in Fig. 7 for the case U04D03F10. A similar qualitative behaviour has been observed for the other cases and thus are not shown here.

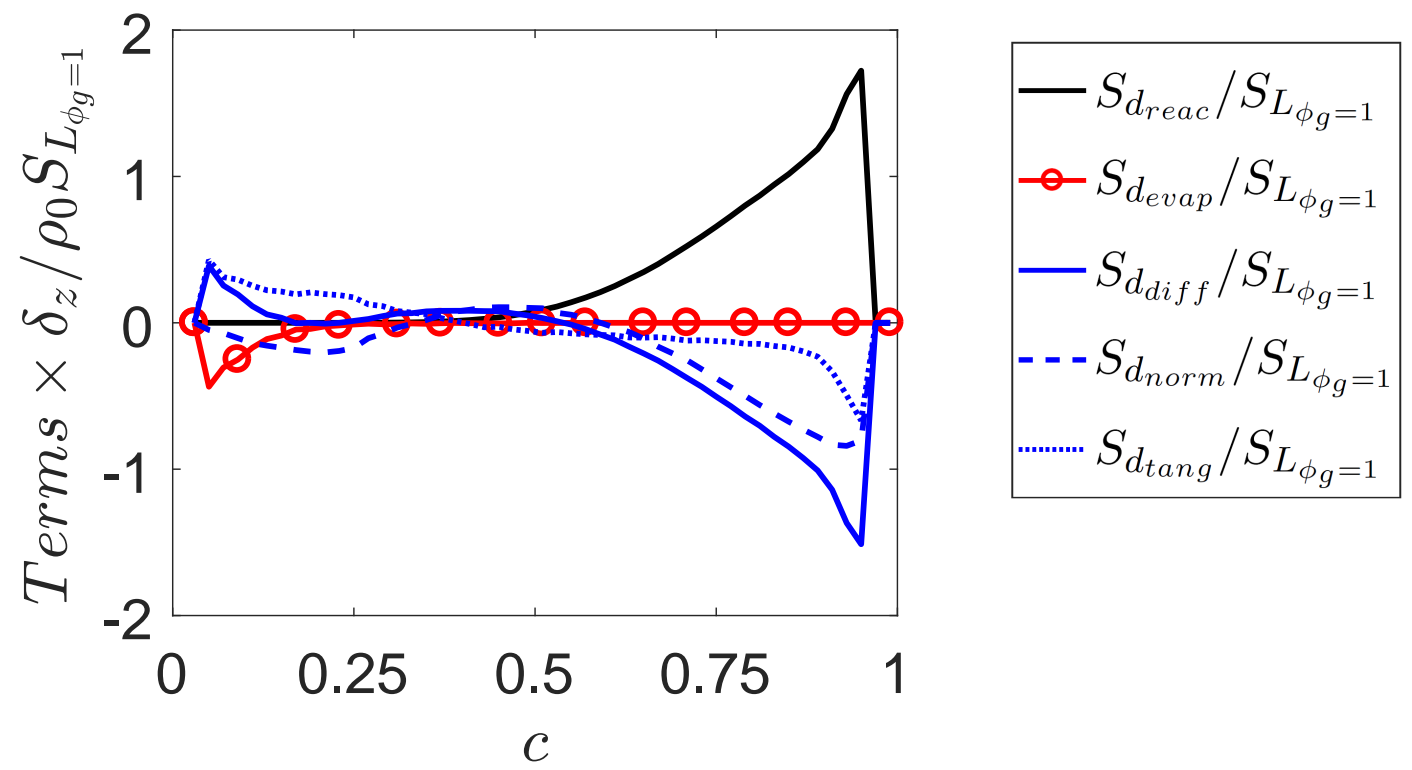

FIG. 7. Variation of the mean values $S_{d_{\text {reac }}} / S_{L_{\phi_{g}=1}}, S_{d_{\text {evap }}} / S_{L_{\phi_{g}=1}}, S_{d_{\text {diff }}} / S_{L_{\phi_{g}=1}}, S_{d_{\text {norm }}} / S_{L_{\phi_{g}=1}}$ and $S_{d_{t a n g}} / S_{L_{\phi_{g}=1}}$ conditional on $c$ on the stoichiometric mixture fraction (i.e. $\xi=\xi_{s t}$ ) isosurface for the case U04D03F10.

The mean values of normal and tangential molecular diffusion components (i.e. $S_{d_{\text {norm }}} / S_{L_{\phi_{g}=1}}$ and $\left.S_{d_{\text {tang }}} / S_{L_{\phi_{g}=1}}\right)$ conditional on $c$ on the stoichiometric mixture fraction (i.e. $\xi=\xi_{s t}$ ) isosurface are also shown in Fig. 7. It can be seen from Fig. 77 that the mean $S_{d_{\text {reac }}} / S_{L_{\phi_{g}=1}}$ assumes positive values with a peak value at around $c=0.8$, whereas the mean values of $S_{d_{d i f f}} / S_{L_{\phi_{g}=1}}$ and $S_{d_{\text {tang }}} / S_{L_{\phi_{g}=1}}$ assume positive values towards the unburned gas side (i.e. $c<0.5)$ and negative values towards the burned gas (i.e. $c>0.5)$ side. This mean 
behaviour for $S_{d_{\text {tang }}} / S_{L_{\phi_{g}=1}}$ originates because of the predominantly negative values of curvature $\kappa_{m, Y}$ for small values of $c$ in the edge flame locations (see Fig. 4 left) but $\kappa_{m, Y}$ assumes predominantly positive values for large values of $c$ for the edge flame locations in this configuration (see Fig. 4 right). The mean normal diffusion component $S_{d_{n_{\text {orm }}}} / S_{L_{\phi_{g}=1}}$ assumes negative values throughout the flame but shows two local minima on the unburned and burned gas sides of the flame. It can further be seen from Fig. 7 that the mean evaporation component $S_{d_{\text {evap }}} / S_{L_{\phi_{g}=1}}$ assumes negligible values in comparison to the other components of $S_{d} / S_{L_{\phi_{g}=1}}$ for the major part of the flame but it assumes non-negligible negative values in the unburned gas side due to strong influences of evaporation, which remain in approximate equilibrium with positive mean values of $S_{d_{d i f f}} / S_{L_{\phi_{g}=1}}$. This further indicates that the mean values of $S_{d_{\text {tang }}} / S_{L_{\phi_{g}=1}}=-2 D \kappa_{m, Y} / S_{L_{\phi_{g}=1}}$ remain large where the mean $S_{d_{\text {evap }}} / S_{L_{\phi_{g}=1}}$ assumes significantly negative values. It is also consistent with the observation from Fig. 4 that the edge flames for small values of $c$ are obtained at the evaporation sites which are associated with negative values of $\kappa_{m, Y}$. The diffusion of freshly evaporated fuel vapour leads to negative mean values of $S_{d_{n o r m}} / S_{L_{\phi_{g}=1}}$ and the associated local minimum on the unburned gas side of the edge flame. This behaviour of the normal diffusion component $S_{d_{n o r m}} / S_{L_{\phi_{g}=1}}$ is different from the previously reported variation of the diffusion component of the edge flame displacement speed in the purely gaseous mixtures, where $S_{d_{n o r m}} / S_{L_{\phi_{g}=1}}$ assumes positive (negative) mean values on the unburned (burned) gas side of the flame $e^{13 / 4}$.

The PDFs of the different components of $S_{d} / S_{L_{\phi_{g}=1}}$ (i.e. $S_{d_{\text {reac }}} / S_{L_{\phi_{g}=1}}, S_{d_{\text {evap }}} / S_{L_{\phi_{g}=1}}$, and $\left.S_{d_{d i f f}} / S_{L_{\phi_{g}=1}}\right)$ are presented in Fig. 8. The PDFs of $S_{d_{\text {evap }}}$ are extremely narrow and exhibit a peak at a slightly negative value for all cases, and its magnitude remains smaller than the magnitude of $S_{d_{d i f f}}$, which remains a major contributor to $S_{d}$ for all cases considered here. It is worth noting that the magnitude of $S_{d_{\text {evap }}}$ remains smaller than the other components of displacement speed and thus the PDFs of $S_{d_{\text {evap }}}$ look like delta functions in Fig. 8 when shown in the x-range for which the variations of other components of displacement speed can be demonstrated clearly. The PDFs of $S_{d_{\text {reac }}} / S_{L_{\phi_{g}=1}}$ show the probability of finding deterministically positive values and show significant probability of finding large values, although the peak value of the PDFs are obtained at a smaller value. The probability of finding large values of temperature (and therefore large magnitudes of $\dot{\omega}_{F}$ ) within the flame kernel increases with decreasing $a_{d}$ due to the greater availability of more reactive mixtures (see Fig. 5). The magnitude of $\nabla Y_{F}$ is also expected to rise for higher values of $\phi_{g}$ in the 

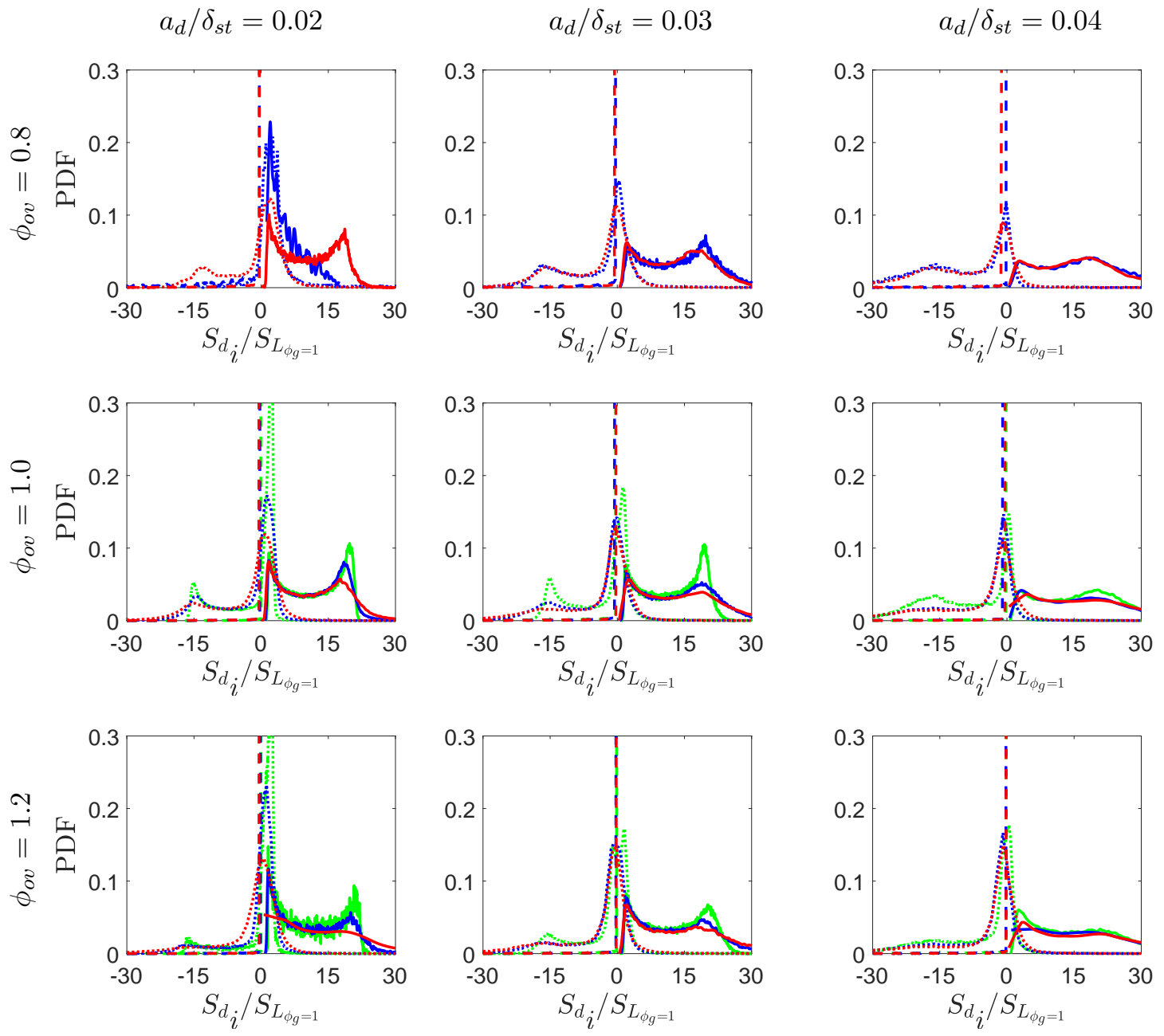

FIG. 8. PDFs of the different components of $S_{d_{i}} / S_{L_{\phi_{g}=1}}$ (i.e. $S_{d_{\text {reac }}} / S_{L_{\phi_{g}=1}}$ (solid line), $S_{d_{\text {evap }}} / S_{L_{\phi_{g}=1}}$ (broken line) and $S_{d_{d i f f}} / S_{L_{\phi_{g}=1}}$ (dotted line) for initial $a_{d} / \delta_{s t}=0.02,0.03$ and 0.04 (left to right) and overall equivalence ratios $\phi_{o v}=0.8,1.0$ and 1.2 (top to bottom), in the case of laminar (green), initial $u^{\prime} / S_{L_{\phi_{g}=1}}=4.0$ (blue) and initial $u^{\prime} / S_{L_{\phi_{g}=1}}=8.0$ (red) conditions. The PDFs of $S_{d_{\text {evap }}} / S_{L_{\phi_{g}=1}}$ and $S_{d_{d i f f}} / S_{L_{\phi_{g}=1}}$ are evaluated using the samples corresponding to $0.1 \leq c \leq 0.9$ on the stoichiometric mixture fraction (i.e. $\xi=\xi_{s t}$ ) isosurface, whereas the PDFs of $S_{d_{\text {reac }}} / S_{L_{\phi_{g}=1}}$ are evaluated using $0.5 \leq c \leq 0.9$ samples on the $\xi=\xi_{s t}$ isosurface.

gaseous phase, and thus higher values of $\left|\nabla Y_{F}\right|$ are more readily available for smaller values of $a_{d}$ for a given value of $\phi_{o v}$. This behaviour strengthens further with increasing $\phi_{o v}$. The relative magnitudes of $\dot{\omega}_{F}$ and $\left|\nabla Y_{F}\right|$ determine the variations of $S_{d_{\text {reac }}} / S_{L_{\phi_{g}=1}}$ (see eqs. 28) in 
response to the changes with $a_{d}$ and $\phi_{o v}$. The turbulent motion affects mixture composition due to its influences on mixing, which affects $\dot{\omega}_{F}$ and $\left|\nabla Y_{F}\right|$, whereas turbulent stretching additionally influences $\left|\nabla Y_{F}\right|$. The relative strengths of these effects ultimately determine the distributions of $S_{d_{\text {reac }}} / S_{L_{\phi_{g}=1}}$ in response to the changes in $u^{\prime} / S_{L_{\phi_{g}=1}}$.

The diffusion component of displacement speed $S_{d_{d i f f}} / S_{L_{\phi_{g}=1}}$ shows both positive and negative values. It has been shown in Fig. 7 that positive (negative) values of $S_{d_{d i f f}} / S_{L_{\phi_{g}=1}}$ are more likely for small (large) values of $c$, and the same qualitative behaviour has been observed for the other cases considered here. The probability of finding negative (positive) values of $S_{d_{d i f f}} / S_{L_{\phi_{g}=1}}$ increases (decreases) with increasing $u^{\prime} / S_{L_{\phi_{g}=1}}$. Moreover, an increase of initial droplet diameter also acts to increase (decrease) the probability of finding negative (positive) values of $S_{d_{d i f f}} / S_{L_{\phi_{g}=1}}$, whereas the qualitative behaviour of the PDFs of $S_{d_{d i f f}} / S_{L_{\phi_{g}=1}}$ for a given droplet diameter remains unchanged for $\phi_{o v}=1.0$ and 1.2 cases. In order to understand the aforementioned behaviours of the PDFs of $S_{d_{d i f f}} / S_{L_{\phi_{g}=1}}$, it is instructive to examine the PDFs of different components of $S_{d_{d i f f}} / S_{L_{\phi_{g}=1}}$ (i.e. $S_{d_{n o r m}} / S_{L_{\phi_{g}=1}}$ and $S_{d_{\text {tang }}} / S_{L_{\phi_{g}=1}}$ ), which are presented in Fig. 9 for all the edge flame cases considered here.

The PDFs of $S_{d_{n o r m}} / S_{L_{\phi_{g}=1}}$ show two peaks at negative values for the cases with initial $a_{d} / \delta_{s t}=0.02$ and 0.03 but this behaviour is not prominent for the $a_{d} / \delta_{s t}=0.04$ cases. The normal diffusion component $S_{d_{n o r m}} / S_{L_{\phi_{g}=1}}$ assumes predominantly negative values and its mean value assumes a local minimum value on the unburned gas side (i.e. $c<0.5$ ), whereas it also shows mostly negative values for $c>0.5$ and its mean value also assumes a local minimum at a value of $c$ for which the maximum value of $S_{d_{n o r m}} / S_{L_{\phi_{g}=1}}$ is obtained (see Fig. 7). This gives rise to the two peaks in the PDFs of $S_{d_{n o r m}} / S_{L_{\phi_{g}=1}}$ observed in the cases with initial $a_{d} / \delta_{s t}=0.02$ and 0.03 , while the relative magnitudes of the peaks depend on the sample sizes from the unburned and burned gas sides of the flame. The PDFs of $c$ on the $\xi=\xi_{s t}$ isosurface are shown in Fig. 10, which reveals that the probability of finding $c>0.5$ within the edge flame for the cases with initial $a_{d} / \delta_{s t}=0.04$ remains much greater than that in the initial $a_{d} / \delta_{s t}=0.02$ and 0.03 cases. In order to explain this behaviour it is worthwhile to consider the PDFs of the modified flame index $\psi$ for the samples corresponding to $0.1 \leq c \leq 0.9$ irrespective of the value of $\xi$, which are shown in Fig. 11 .

It can be seen from Fig. 11 that the probability of finding $\psi=0$ increases with increasing $a_{d}$ due to the slow evaporation rate for large droplets that gives rise to imperfect mixing, which in turn increases the extent of the non-premixed mode of burning at $\xi=\xi_{s t}$. It can 

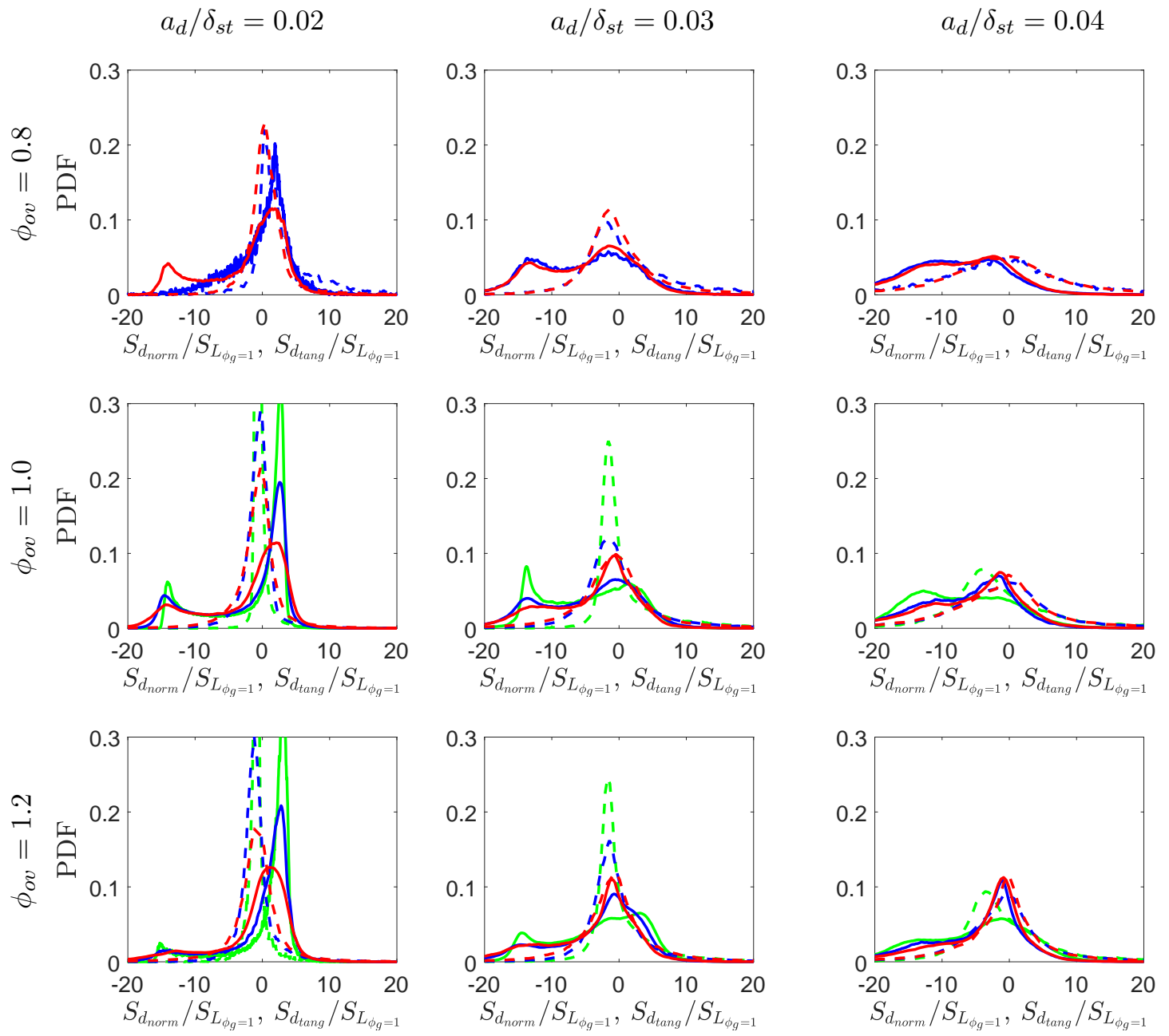

FIG. 9. PDFs of the different components of $S_{d_{d i f f}} / S_{L_{\phi_{g}=1}}$ (i.e. $S_{d_{n o r m}} / S_{L_{\phi_{g}=1}}$ (solid line) and $S_{d_{\text {tang }}} / S_{L_{\phi_{g}=1}}$ (broken line)) based on the samples corresponding to $0.1 \leq c \leq 0.9$ on the stoichiometric mixture fraction (i.e. $\xi=\xi_{s t}$ ) isosurface for initial $a_{d} / \delta_{s t}=0.02,0.03$ and 0.04 (left to right) and overall equivalence ratios $\phi_{o v}=0.8,1.0$ and 1.2 (top to bottom), in the case of laminar (green), initial $u^{\prime} / S_{L_{\phi_{g}=1}}=4.0$ (blue) and initial $u^{\prime} / S_{L_{\phi_{g}=1}}=8.0$ (red) conditions.

further be seen from Fig. 11 that fuel-lean premixed modes of burning are predominantly obtained for $\phi_{o v}=0.8$ and 1.0 and the occurrence of fuel-rich premixed flame becomes increasingly prominent with increasing $a_{d}$. This is consistent with the predominance of $\phi_{g}<$ $\phi_{o v}$ in the $\phi_{o v}=0.8$ and 1.0, as demonstrated in Fig. 5. In the case of $\phi_{o v}=1.2$, rich premixed combustion modes are predominantly obtained for initial $a_{d} / \delta_{s t}=0.02$ but the probability of 

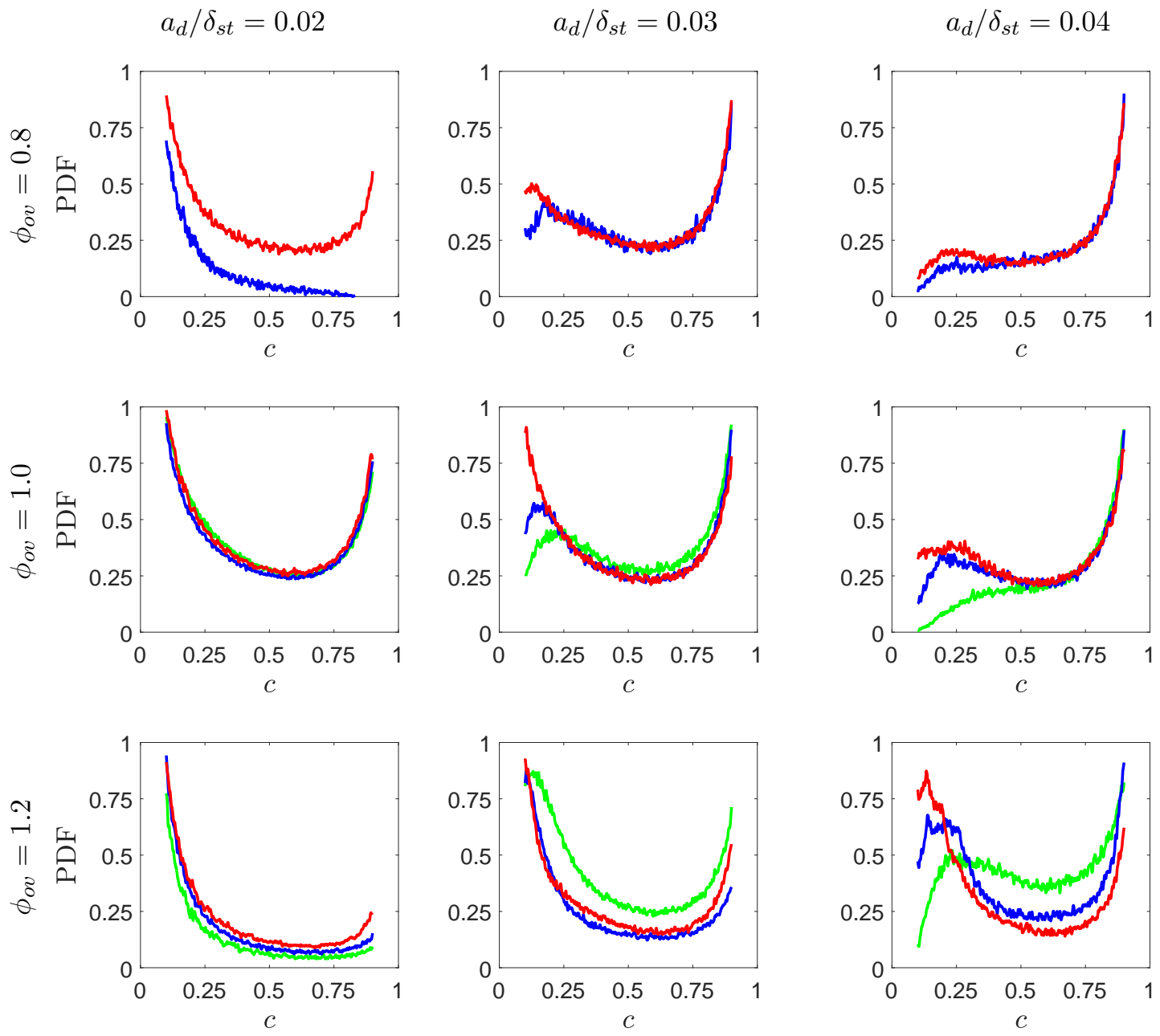

FIG. 10. PDFs of $c$ on the $\xi=\xi_{s t}$ isosurface for initial $a_{d} / \delta_{s t}=0.02,0.03$ and 0.04 (left to right) and overall equivalence ratios $\phi_{o v}=0.8,1.0$ and 1.2 (top to bottom), in the case of laminar (green), initial $u^{\prime} / S_{L_{\phi_{g}=1}}=4.0$ (blue) and initial $u^{\prime} / S_{L_{\phi_{g}=1}}=8.0$ (red) conditions.

finding both diffusion and fuel-lean premixed flames increases with increasing droplet size. The highest values of burning rate and burned gas temperature are obtained around the stoichiometric mixture and thus, the strengthening of diffusion flame mode combustion for large droplets is reflected in the higher probability of finding large values of $c$ (i.e. $c>0.5$ ) for cases with $a_{d} / \delta_{s t}=0.04$ (see Fig. 10).

As the samples in the $a_{d} / \delta_{s t}=0.04$ usually include significantly higher burned gas contribution (i.e. $c>0.5$ ) than that from the unburned gas side (i.e. $c<0.5$ ), thus only one 

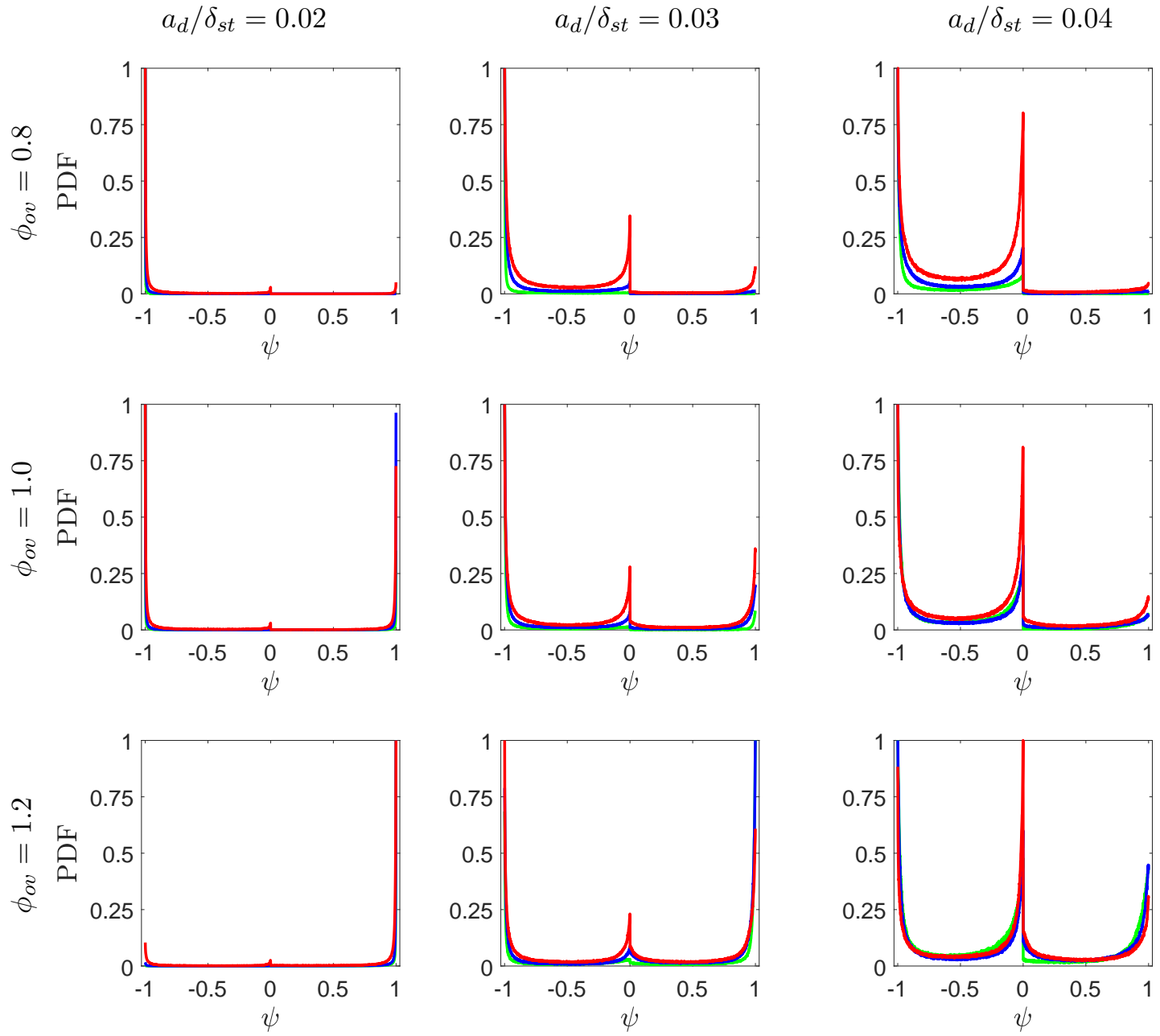

FIG. 11. PDFs of flame index $\psi$ for the samples corresponding to $0.1 \leq c \leq 0.9$ for initial $a_{d} / \delta_{s t}=0.02,0.03$ and 0.04 (left to right) and overall equivalence ratios $\phi_{o v}=0.8,1.0$ and 1.2 (top to bottom), in the case of laminar (green), initial $u^{\prime} / S_{L_{\phi_{g}=1}}=4.0$ (blue) and initial $u^{\prime} / S_{L_{\phi_{g}=1}}=8.0$ (red) conditions.

of the peaks is prominent in the $S_{d_{\text {norm }}} / S_{L_{\phi_{g}=1}}$ PDF in this case. By contrast, the samples in the initial $a_{d} / \delta_{s t}=0.02$ and 0.03 cases include sizeable proportions from both unburned and burned gases and thus two peaks are obtained for the $S_{d_{n o r m}} / S_{L_{\phi_{g}=1}}$ PDFs. An increase in $u^{\prime} / S_{L_{\phi_{g}=1}}$ influences the distribution of $\phi_{g}$, which affects the magnitudes of $\left|\nabla Y_{F}\right|$ and $|\nabla| \nabla Y_{F}||$, which also increase with increasing turbulence intensity. The combined effects of these mechanisms determine the $u^{\prime} / S_{L_{\phi_{g}=1}}$ dependences of $S_{d_{\text {norm }}} / S_{L_{\phi_{g}=1}}$ and it has been found that the values at which positive and negative peaks of the $S_{d_{n o r m}} / S_{L_{\phi_{g}=1}}$ PDFs are 
obtained are not significantly affected by $u^{\prime} / S_{L_{\phi_{g}=1}}$. However, higher magnitudes of $\left|\nabla Y_{F}\right|$ and $|\partial| \nabla Y_{F}\left|/ \partial x_{j}\right|$ are likely in the highly reactive mixtures with $\phi_{g} \approx 1.0$ within the flame front, which are more readily obtained for small droplets, and thus the peaks of $S_{d_{n o r m}} / S_{L_{\phi_{g}=1}}$ PDFs are sharper in the initial $a_{d} / \delta_{s t}=0.02$ cases than in the cases with initial $a_{d} / \delta_{s t}=0.03$ and 0.04 .

The tangential diffusion component $S_{d_{\text {tang }}} / S_{L_{\phi_{g}=1}}=-2 D \kappa_{m, Y} / S_{L_{\phi_{g}=1}}$ exhibits both positive and negative values. The width of $S_{d_{t a n g}} / S_{L_{\phi_{g}=1}}$ increases with increasing $u^{\prime} / S_{L_{\phi_{g}=1}}$ as the range of the curvature $\kappa_{m, Y}$ of the fuel mass fraction isosurface increases due to greater extent of flame wrinkling. The flame-droplet interaction gives rise to flame wrinkling, which leads to a range of $\kappa_{m, Y}$ and $S_{d_{\text {tang }}} / S_{L_{\phi_{g}=1}}=-2 D \kappa_{m, Y} / S_{L_{\phi_{g}=1}}$ values even in the laminar flames. As the droplet-induced flame wrinkling strengthens with increasing $a_{d}$ and $\phi_{o v}$, the width of $S_{d_{\text {tang }}} / S_{L_{\phi_{g}=1}}$ PDFs also increases. The droplet-induced flame wrinkling is eclipsed by turbulence-induced flame wrinkling with increasing $u^{\prime} / S_{L_{\phi_{g}=1}}$ 47748153154 (see Figs. 22 3) and thus the widening of the $S_{d_{\text {tang }}} / S_{L_{\phi_{g}=1}}$ PDFs with increasing $a_{d}$ and $\phi_{o v}$ is not prominent in the turbulent cases.

Furthermore, an increase in $u^{\prime} / S_{L_{\phi_{g}=1}}$ leads to a greater amount of heat transfer from the hot gas kernel and thus the growth rate of the flame kernels decreases with increasing $u^{\prime} / S_{L_{\phi_{q}=1}}$. This can be substantiated from the temporal evolution of the normalised burned gas volume $V_{c \geq 0.9} / V_{s p}$ (where $V_{s p}=4 \pi R_{s p}^{3} / 3$ is the energy deposition volume) of the region with $c \geq 0.9$, which is shown in Fig. 12 . This is reflected in an increased probability of finding large negative values of $S_{d_{t a n g}} / S_{L_{\phi_{g}=1}}$ with increasing $u^{\prime} / S_{L_{\phi_{g}=1}}$. A comparison between Figs. 7 and 8 reveals that the higher probability of finding large negative values of $S_{d_{\text {tang }}} / S_{L_{\phi_{g}=1}}$ is principally responsible for the increases in the probability of finding negative values of $S_{d_{d i f f}} / S_{L_{\phi_{g}=1}}$ with increasing $u^{\prime} / S_{L_{\phi_{g}=1}}$. The greater availability of highly reactive mixture with $\phi_{g}$ close to unity within the flame front for smaller droplet diameters (see Fig. 5) leads to an increasing extent of burning (i.e. increased volume of $V_{c \geq 0.9} / V_{s p}$ ) with decreasing droplet diameter for the $\phi_{o v}=1.0$ and 1.2 cases. By contrast, $V_{c \geq 0.9} / V_{s p}$ remains comparable for different droplet diameters and exhibits a mild increase with increasing $a_{d} / \delta_{s t}$ in the case of $\phi_{o v}=0.8$, as the local mixture inhomogeneities induced by the slow evaporation of large droplets increases the probability of finding local islands of more reactive mixtures than for $\phi_{g}=0.8$. Thus, the probability of finding negative values of $S_{d_{t a n g}} / S_{L_{\phi_{g}=1}}$ increases with increasing (decreasing) $a_{d} / \delta_{s t}$ for $\phi_{o v}=1.0$ and $1.2\left(\phi_{o v}=0.8\right)$ cases. Moreover, $V_{c \geq 0.9} / V_{s p}$ 

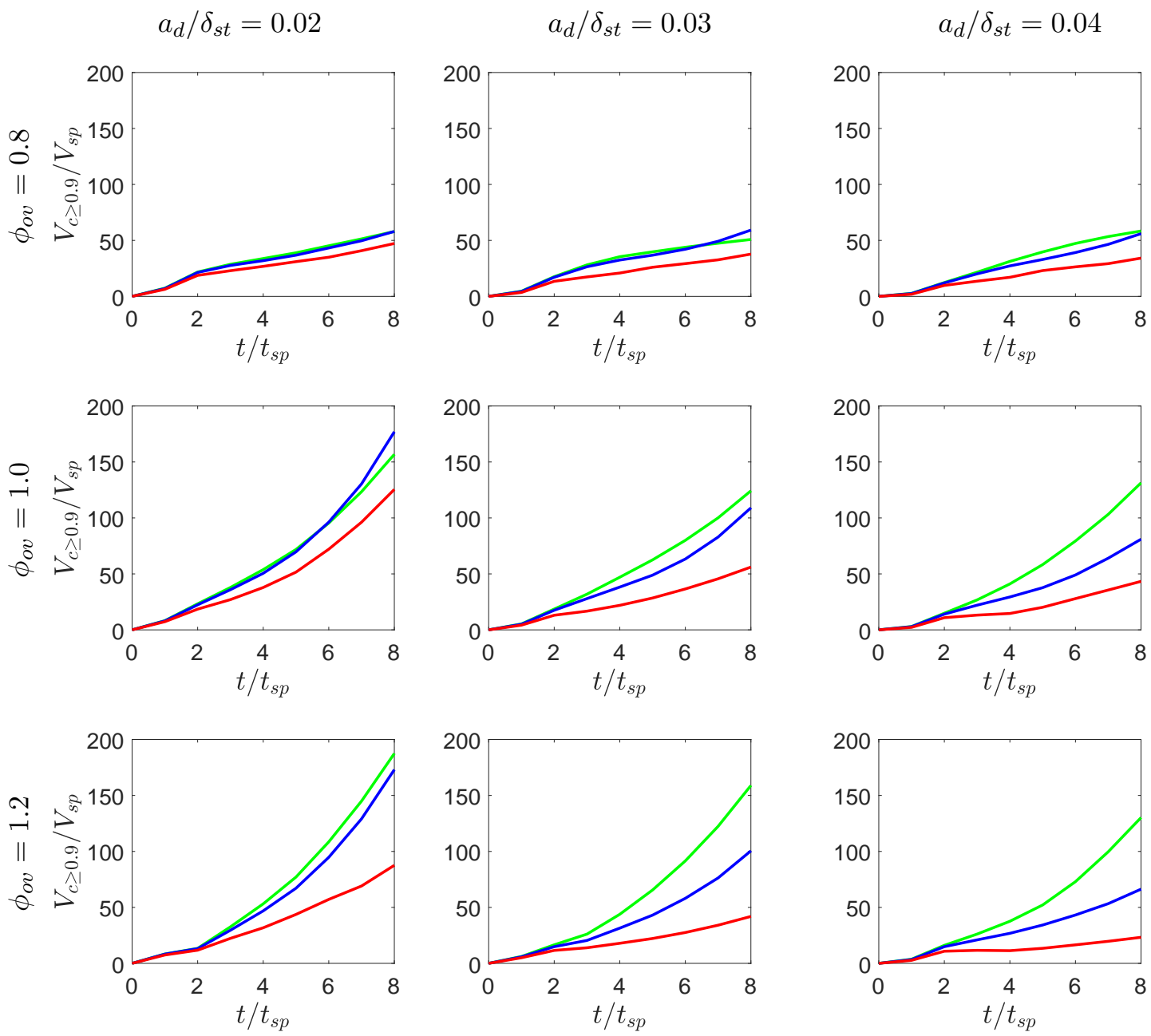

FIG. 12. Temporal evolutions of the normalised burned gas volume $V_{c \geq 0.9} / V_{s p}$ of the region corresponding to $c \geq 0.9$ for initial $a_{d} / \delta_{s t}=0.02,0.03$ and 0.04 (left to right) and overall equivalence ratios $\phi_{o v}=0.8,1.0$ and 1.2 (top to bottom), in the case of laminar (green), initial $u^{\prime} / S_{L_{\phi_{g}=1}}=4.0$ (blue) and initial $u^{\prime} / S_{L_{\phi_{g}=1}}=8.0$ (red) conditions.

increases with increasing $\phi_{o v}$ for a given set of values of $u^{\prime} / S_{L_{\phi_{g}=1}}$ and $a_{d} / \delta_{s t}$, which is reflected in the higher probability of finding negative values of $S_{d_{t a n g}} / S_{L_{\phi_{g}=1}}$ for smaller values of $\phi_{o v}$.

A comparison between Figs. 6]8 and 9 reveals that the negative value of the edge flame speed $S_{e}$ arises predominantly due to the diffusion component of displacement speed, $S_{d_{d i f f}}$. In particular, the predominantly negative values of the normal diffusion component $S_{d_{\text {norm }}}$ 
lead to local negative values of $S_{e}$, when it overcomes the positive contributions arising from other components (e.g. $S_{d_{\text {reac }}}$ and for positive values of $S_{d_{\text {tang }}}$ ). However, in the locations where $S_{d_{\text {tang }}}$ is negative, it aids the normal diffusion component and a negative value of $S_{e}$ is obtained when $S_{d_{\text {diff }}}=\left(S_{d_{\text {norm }}}+S_{d_{\text {tang }}}\right)$ overcomes $S_{d_{\text {reac }}}$.

Figures 6, 8 and 9 further suggest that the droplet evaporation does not affect the edge flame speed significantly through the component $S_{d_{\text {evap }}}$ but the evaporation characteristics affect the equivalence ratio distribution in the gaseous phase, which in turn affects the reaction rate and scalar gradient magnitude (i.e. $\dot{\omega}_{F}$ and $\left|\nabla Y_{F}\right|$ ) and these quantities eventually affect the statistics of $S_{e}$ through the displacement speed components $S_{d_{\text {reac }}}$ and $S_{d_{\text {norm }}}$.

\section{Local curvature, strain rate and scalar gradient dependences of edge flame speed}

Finally, it is worthwhile to consider the influences of strain rate, curvature and scalar gradient on the local behaviours of the edge flame speed $S_{e}$. The mean values of $S_{e} / S_{L_{\phi_{g}=1}}$ conditional on $\kappa_{m, Y} \times \delta_{z}$ are shown in Fig. 13. It is evident from Fig. 13 that the $\kappa_{m, Y} \times \delta_{z}$ dependence of $S_{e} / S_{L_{\phi_{g}=1}}$ is non-linear in nature. For the $\phi_{o v}=0.8$ and 1.0 cases with small initial droplet diameter, the edge flame speed does not vary significantly for negative and small positive values of $\kappa_{m, Y}$ followed by a decreasing trend with increasing $\kappa_{m, Y}$ for positive curvatures (i.e $\kappa_{m, Y}>0$ ). By contrast, for the cases with $\phi_{o v}=1.2$ the corresponding curvature dependence of edge flame speed remains weak for the whole range of curvature $\kappa_{m, Y} \times \delta_{z}$. There is also a weak increasing trend of the mean values of $S_{e} / S_{L_{\phi_{g}=1}}$ with $\kappa_{m, Y} \times \delta_{z}$ for the cases U08D02F12 and U00D03F12. It has already been demonstrated that the statistical behaviour of $S_{e}$ is governed by $S_{d}$ (see Fig. 6). The non-linear $\kappa_{m, Y}$ dependence of $S_{d}$ and the positive correlating branch in some cases have been reported for edge flames in purely gaseous fuel-air mixtures in previous analyses $13 / 4|7| 18|25| 26$. The negative correlation trend between $S_{d}$ and $\kappa_{m, Y}$ originates principally due to the tangential diffusion component of displacement speed $S_{d_{\text {tang }}} / S_{L_{\phi_{g}=1}}=-2 D \kappa_{m, Y}$, whereas the combined reaction and normal diffusion component $\left(S_{d_{\text {reac }}}+S_{d_{\text {norm }}}\right) / S_{L_{\phi_{g}=1}}$ induces non-linearity in the correlation $\frac{13 / 4 \mid 18}{12}$ and the same is valid here and thus not shown for the sake of brevity.

Several previous analyses demonstrated that the net correlation between $S_{d_{\text {reac }}} / S_{L_{\phi_{g}=1}}$ and $\kappa_{m, Y}$ remains positive for statistically spherical premixed $\sqrt[56]{58}$ and partially-premixed $\frac{13|4| 18}{13}$ 

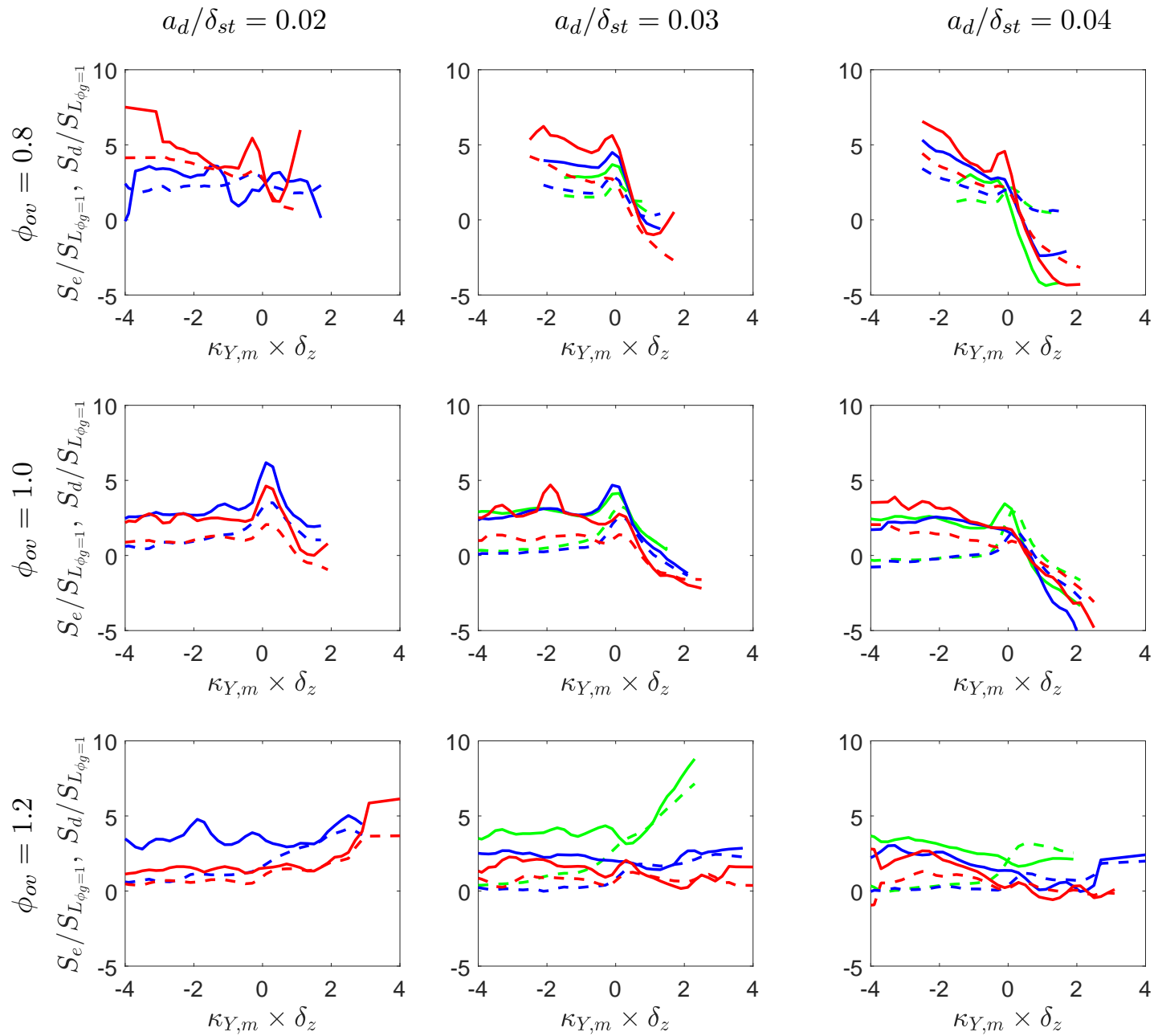

FIG. 13. Variations of the mean values of $S_{e} / S_{L_{\phi_{g}=1}}$ (solid line) and $S_{d} / S_{L_{\phi_{g}=1}}$ conditional on $\kappa_{m, Y} \times \delta_{z}$ for the samples corresponding to $0.1 \leq c \leq 0.9$ on the $\xi=\xi_{s t}$ isosurface for initial $a_{d} / \delta_{s t}=0.02,0.03$ and 0.04 (left to right) and overall equivalence ratios $\phi_{o v}=0.8,1.0$ and 1.2 (top to bottom), in the case of laminar (green), initial $u^{\prime} / S_{L_{\phi_{g}=1}}=4.0$ (blue) and initial $u^{\prime} / S_{L_{\phi_{g}=1}}=8.0$ (red) conditions.

flames and similar qualitative behaviour has been observed here (not shown). The relative strengths of the positive correlation between $\left(S_{d_{\text {reac }}}+S_{d_{n o r m}}\right) / S_{L_{\phi_{g}=1}}$ and $\kappa_{m, Y}$, and deterministically negative correlation between $S_{d_{\text {tang }}}$ and $\kappa_{m, Y}$ lead to the net curvature dependences of $S_{d}$, which eventually determines the $\kappa_{m, Y}$ dependence of $S_{e}$. The strength of the positive correlation between $\left(S_{d_{\text {reac }}}+S_{d_{n o r m}}\right) / S_{L_{\phi_{g}=1}}$ and $\kappa_{m, Y}$ depends on the mixture composition 
and mean flame radius. However, the positive correlation between $\left(S_{d_{\text {reac }}}+S_{d_{\text {norm }}}\right) / S_{L_{\phi_{g}=1}}$ and $\kappa_{m, Y}$ weakens with increasing mean flame radius ${ }^{56} \sqrt[58]{ }$ (not shown here but the qualitative behaviour is similar).

It can be seen from Fig. 12 that $V_{c \geq 0.9} / V_{s p}$ for the U08D02F12 case is smaller than other cases with $\phi_{o v}=1.2$ and $a_{d} / \delta_{s t}=0.02$. Similarly, $V_{c \geq 0.9} / V_{s p}$ decreases with increasing $a_{d} / \delta_{s t}$ for $\phi_{o v}=1.2$. An augmented heat transfer rate for high values of $u^{\prime} / S_{L_{\phi_{g}=1}}$ gives rise to a smaller flame kernel in the U08D02F12 case than in the other cases with $\phi_{o v}=1.2$ and $a_{d} / \delta_{s t}=0.02$. In the U00D03F12 case, slow evaporation of large droplets leads to localised fuel-rich islands and the evaporated gaseous fuel does not sufficiently mix with the surrounding air to produce highly reactive mixture with $\phi_{g} \approx 1.0$ in the absence of turbulence. This leads to weak burning and accordingly smaller values of $V_{c \geq 0.9} / V_{s p}$ in this case than that in the laminar case with $\phi_{o v}=1.2$ and initial $a_{d} / \delta_{s t}=0.02$. It is worth noting that the evaporation rate remains small even for the laminar cases with $\phi_{o v}=0.8$ and 1.0 for large droplet sizes, but the lack of sufficient mixing produces a higher probability of finding a more reactive mixture due to the smaller number density of droplets in the corresponding $\phi_{o v}=0.8$ cases than in the corresponding $\phi_{o v}=1.2$ cases. However the greater number density of droplets in the $\phi_{o v}=1.2$ cases leads to higher probabilities of finding a weakly combustible fuel-rich mixture than in the corresponding $\phi_{o v}=1.0$ cases. Accordingly $V_{c \geq 0.9} / V_{s p}$ in the laminar case with initial $a_{d} / \delta_{s t}=0.04$ remains smaller for $\phi_{o v}=0.8$ in comparison to the corresponding $\phi_{o v}=1.0$ and 1.2 cases. The smaller mean flame radius in the U08D02F12 case than in other cases with $\phi_{o v}=1.2$ and initial $a_{d} / \delta_{s t}=$ 0.02 , and similarly smaller flame kernel size in the U00D03F12 case than in the laminar $\phi_{o v}=1.2$ case with initial $a_{d} / \delta_{s t}=0.02$, leads to relatively strong positive correlations between $\left(S_{d_{\text {reac }}}+S_{d_{n o r m}}\right)$ and $\kappa_{Y, m}$, which overcome the negative correlation between $S_{d_{\text {tang }}}$ and $\kappa_{Y, m}$ to give rise to a weak increasing trend of the mean value of $S_{d}$ conditioned upon $\kappa_{Y, m}$ in cases U08D02F12 and U00D03F12 (see Fig. 13). Although the burned gas radius for the laminar $\phi_{o v}=1.2$ case with initial $a_{d} / \delta_{s t}=0.04$ remains smaller than that in the U00D03F12 case, the availability of highly reactive mixture with $\phi_{g} \approx 1.0$ in the initial $a_{d} / \delta_{s t}=0.04$ case is much smaller due to slower evaporation rate and thus the effects of $S_{d_{\text {reac }}}$ in the initial $a_{d} / \delta_{s t}=0.04$ case are weaker than in the $a_{d} / \delta_{s t}=0.03$ case for $\phi_{o v}=1.2$. Therefore, the positive correlation between $\left(S_{d_{\text {reac }}}+S_{d_{n o r m}}\right)$ and $\kappa_{Y, m}$ almost nullifies the negative correlation between $S_{d_{\text {reac }}}$ and $\kappa_{Y, m}$ in the U00D4F12 case to yield a 
weak curvature dependence of the edge flame speed.

It has already been shown in Figs. 5 and 11 that the probability of fuel-lean combustion increases with increasing $a_{d} / \delta_{s t}$. Thus, the characteristic values of $u^{\prime} / S_{L_{\phi_{g}=1}}$ under which combustion takes place for large droplets remain relatively high. It is well-known $\frac{59}{61}$ that the curvature dependence of $S_{d}$ for a given equivalence ratio $\phi_{g}$ becomes weak for large $u^{\prime} / S_{L_{\phi_{g}}}$ and accordingly the curvature dependences of $S_{d}$ and $S_{e}$ become progressively weak with increasing droplet diameter.

The mean values of $S_{e} / S_{L_{\phi_{g}=1}}$ and $S_{d} / S_{L_{\phi_{g}=1}}$ conditional on $a_{T, Y} \times \delta_{s t} / S_{L_{\phi_{g}=1}}$ are shown in Fig. 14, which reveals mainly a positive correlation, while the correlation trends for the laminar cases are not clear because of the limited variation of $a_{T, Y}$ values in these flames. The $a_{T, Y}$ dependence of $S_{e}$ is also governed by the tangential strain rate dependence of the displacement speed. In all cases $\left(S_{d_{\text {reac }}}+S_{d_{\text {norm }}}\right)$ remains negatively correlated with $a_{T, Y}$, whereas $S_{d_{\text {tang }}}$ and $a_{T, Y}$ are found to be positively correlated with each other due to negative correlation between $a_{T, Y}$ and $\kappa_{m, Y}$ (not shown here). These correlations are consistent with previous findings for gaseous phase combustion $\frac{1 / 3 / 456}{58}$ and the physical explanations behind this behaviour have been provided elsewhere $e^{1 / 3 / 456} \sqrt[58]{5}$ and thus are not repeated here. In the cases considered here, the positive correlation between $S_{d_{\text {tang }}}$ and $a_{T, Y}$ dominates over the negative correlation between $\left(S_{d_{\text {reac }}}+S_{d_{\text {norm }}}\right)$ and $a_{T, Y}$ to yield a net positive correlation between $S_{d}$ conditional on $a_{T, Y}$, which in turn leads to a positive correlation between $S_{e}$ and $a_{T, Y}$

It has been found that an increase in $a_{T, Y}$ gives rise to an increase in $\left|\nabla Y_{F}\right|$, whereas $\left|\nabla Y_{F}\right|$ is non-linearly related to $\kappa_{m, Y}$ for all cases considered here (not shown here) and the physical explanations behind this behaviour were provided in Refs. 13 [1/56 58 , and are not repeated here. The combination of positive correlation between $a_{T, Y}$ and $\left|\nabla Y_{F}\right|$ and nonlinear curvature $\kappa_{m, Y}$ dependence of $\left|\nabla Y_{F}\right|$ leads to a non-monotonic $\left|\nabla Y_{F}\right|$ dependence of $S_{e}$ so that both positive and negative correlating branches can be observed from Fig. 15. This non-monotonic $\left|\nabla Y_{F}\right|$ dependence of $S_{e}$ has been found to be consistent with previous findings for purely gaseous edge flames $1 \frac{131417}{}$.

The gradients $\left|\nabla Y_{F}\right|$ and $|\nabla \xi|$ remain predominantly positively correlated due to the diffusion mode of burning in the edge flame ${ }^{1 / 3 / 4}$ but a weak negative correlation between $\left|\nabla Y_{F}\right|$ and $|\nabla \xi|$ is observed due to the contribution of the premixed mode of combustion. This can be substantiated from Fig. 16 where mean values of $\left|\nabla Y_{F}\right| \times \delta_{z}$ conditional on 

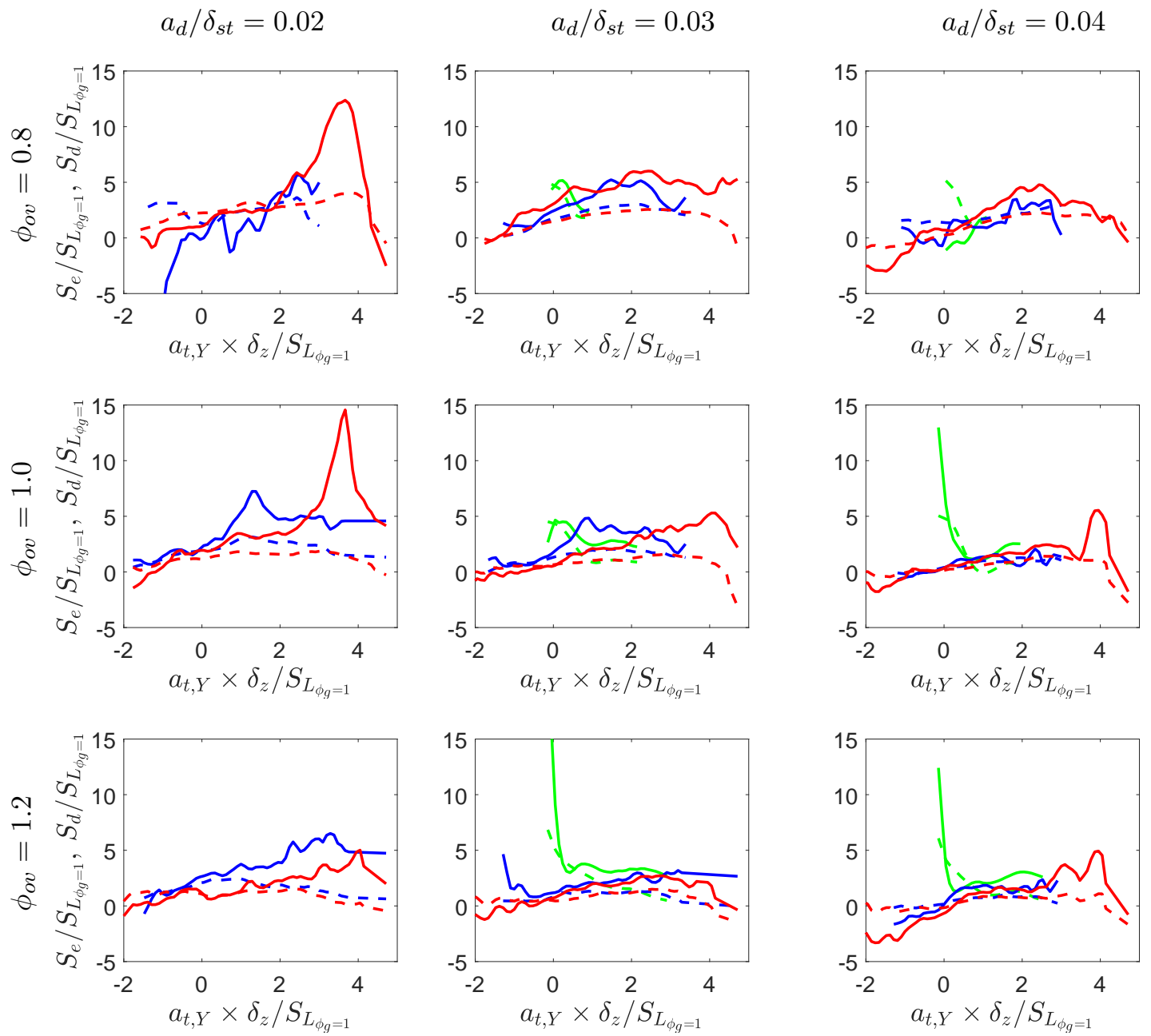

FIG. 14. Variations of the mean values of $S_{e} / S_{L_{\phi_{g}=1}}$ (solid line) and $S_{d} / S_{L_{\phi_{g}=1}}$ conditional on $a_{T, Y} \times \delta_{z} / S_{L_{\phi_{g}=1}}$ for the samples corresponding to $0.1 \leq c \leq 0.9$ on the $\xi=\xi_{s t}$ isosurface for initial $a_{d} / \delta_{s t}=0.02,0.03$ and 0.04 (left to right) and overall equivalence ratios $\phi_{o v}=0.8,1.0$ and 1.2 (top to bottom), in the case of laminar (green), initial $u^{\prime} / S_{L_{\phi_{g}=1}}=4.0$ (blue) and initial $u^{\prime} / S_{L_{\phi_{g}=1}}=8.0$ (red) conditions.

$|\nabla \xi| \times \delta_{z}$ are shown for all cases where the edge flame behaviour is observed. The combination of the non-linear $\left|\nabla Y_{F}\right|$ dependence of $S_{e}$ and predominantly positive correlating branch between $\left|\nabla Y_{F}\right|$ and $|\nabla \xi|$ gives rise to a non-linear $|\nabla \xi|$ dependence of the edge flame speed where both positive and negative correlation trends have been observed, as can be confirmed from Fig. 17. This non-monotonic $|\nabla \xi|$ dependence of $S_{e}$ for the cases considered has been found to be consistent with previous findings for edge flames in purely gaseous 

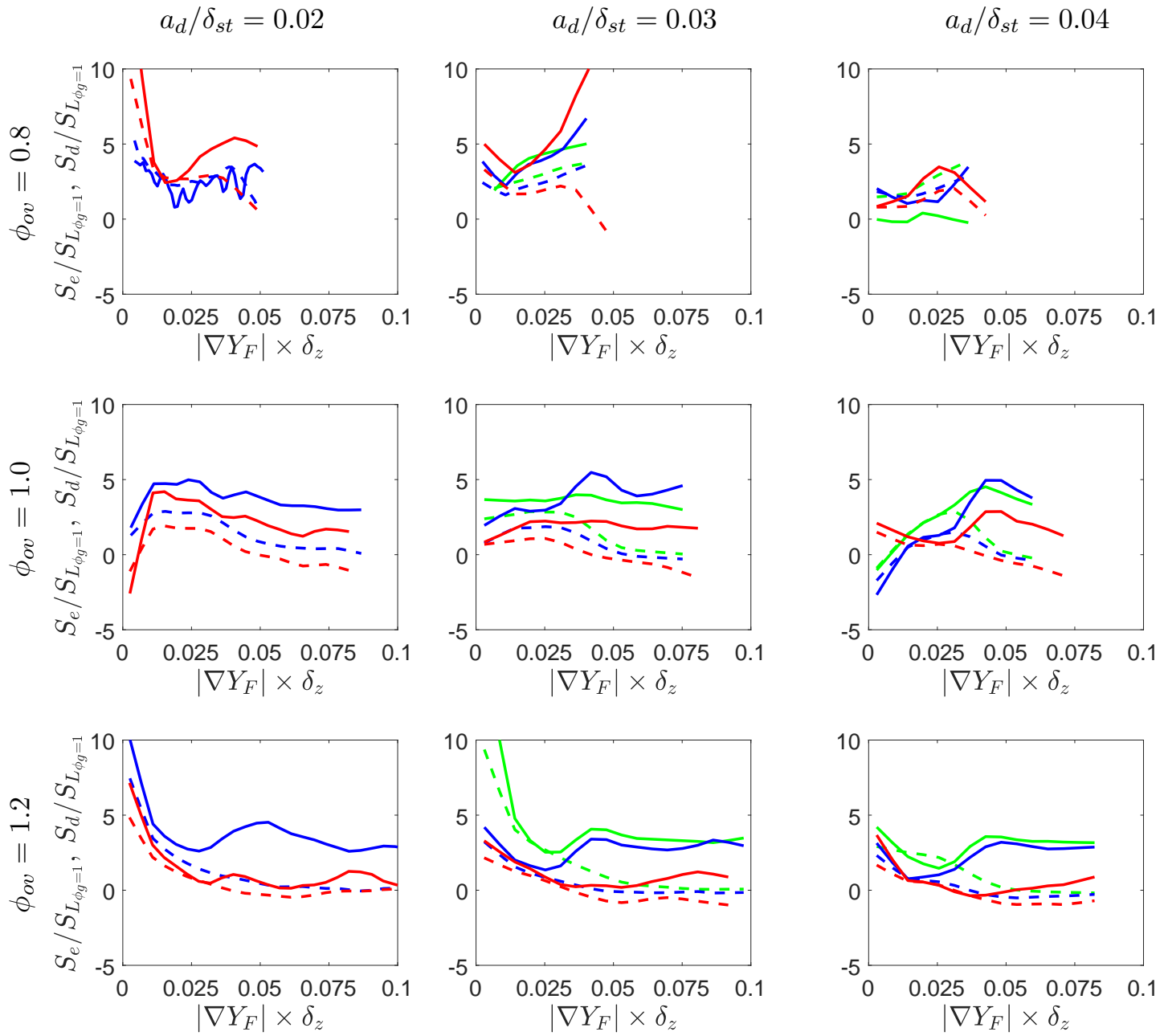

FIG. 15. Variations of the mean values of $S_{e} / S_{L_{\phi_{g}=1}}$ (solid line) and $S_{d} / S_{L_{\phi_{g}=1}}$ conditional on $\left|\nabla Y_{F}\right| \times \delta_{z}$ for the samples corresponding to $0.1 \leq c \leq 0.9$ on the $\xi=\xi_{s t}$ isosurface for initial $a_{d} / \delta_{s t}=0.02,0.03$ and 0.04 (left to right) and overall equivalence ratios $\phi_{o v}=0.8,1.0$ and 1.2 (top to bottom), in the case of laminar (green), initial $u^{\prime} / S_{L_{\phi_{g}=1}}=4.0$ (blue) and initial $u^{\prime} / S_{L_{\phi_{g}=1}}=8.0$ (red) conditions.

phase $\frac{1|3| 4|7| 9|10| 12|18| 21|25| 26}{\text {. }}$

It can be seen from Fig 13 and Fig 14 that the curvature $\left(\kappa_{m, Y}\right)$ and tangential strain rate $\left(a_{T, Y}\right)$ dependences of $S_{e}$ and $S_{d}$ (also for $S_{d}^{*}$, which has been checked but not shown here for brevity) are qualitatively similar. This suggests that $S_{Z}^{*}$ does not significantly affect the local curvature and strain rate dependences of edge flame speed $S_{e}$. However, $\left|\nabla Y_{F}\right|$ and $|\nabla \xi|$ dependences of $S_{e}$ and $S_{d}$ are significantly different (see Figs. 15 and 17) and this 

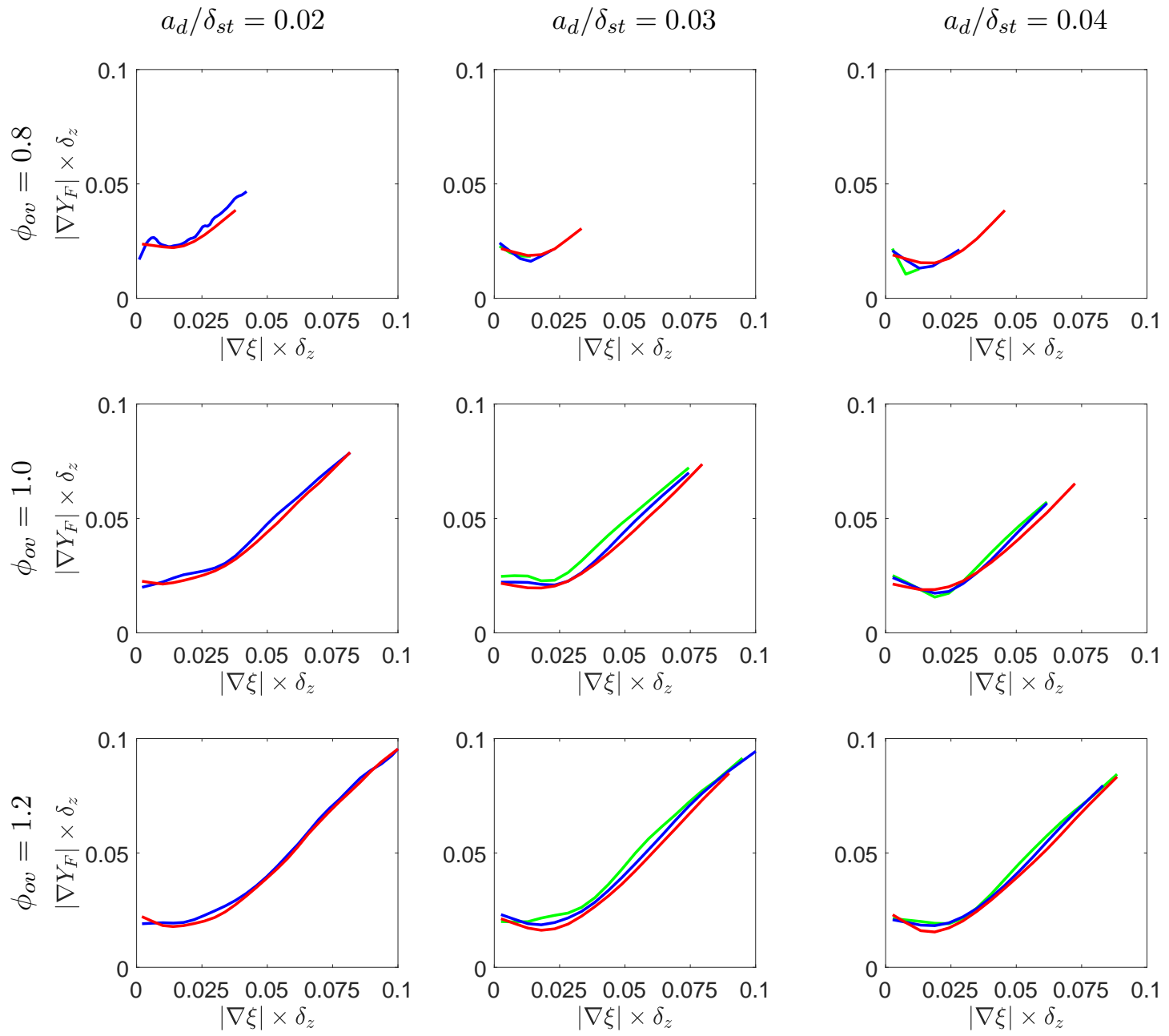

FIG. 16. Variations of the mean values of $\left|\nabla Y_{F}\right| \times \delta_{z}$ conditional on $|\nabla \xi| \times \delta_{z}$ for the samples corresponding to $0.1 \leq c \leq 0.9$ on the $\xi=\xi_{s t}$ isosurface for initial $a_{d} / \delta_{s t}=0.02,0.03$ and 0.04 (left to right) and overall equivalence ratios $\phi_{o v}=0.8,1.0$ and 1.2 (top to bottom), in the case of laminar (green), initial $u^{\prime} / S_{L_{\phi_{g}=1}}=4.0$ (blue) and initial $u^{\prime} / S_{L_{\phi_{g}=1}}=8.0$ (red) conditions.

behaviour becomes increasingly evident for large droplet cases (e.g. initial $a_{d} / \delta_{s t}=0.04$ cases) where the effects of mixture fraction gradients are relatively strong due to local mixture inhomogeneity resulting from a slow evaporation rate. As $\left|\nabla Y_{F}\right|$ and $|\nabla \xi|$ are interrelated, and $|\nabla \xi|$ affects $S_{Z}^{*}$ and its components (see eqs. 27.33), the scalar gradient dependences of $S_{e}$ are dependent not only by $S_{d}$ but also by $S_{Z}^{*}$. Thus, the displacement speed of mixture fraction isosurface can potentially play an important role in determining 
the edge flame $S_{e}$ behaviour in response to the changes in $\left|\nabla Y_{F}\right|$ and $|\nabla \xi|$.
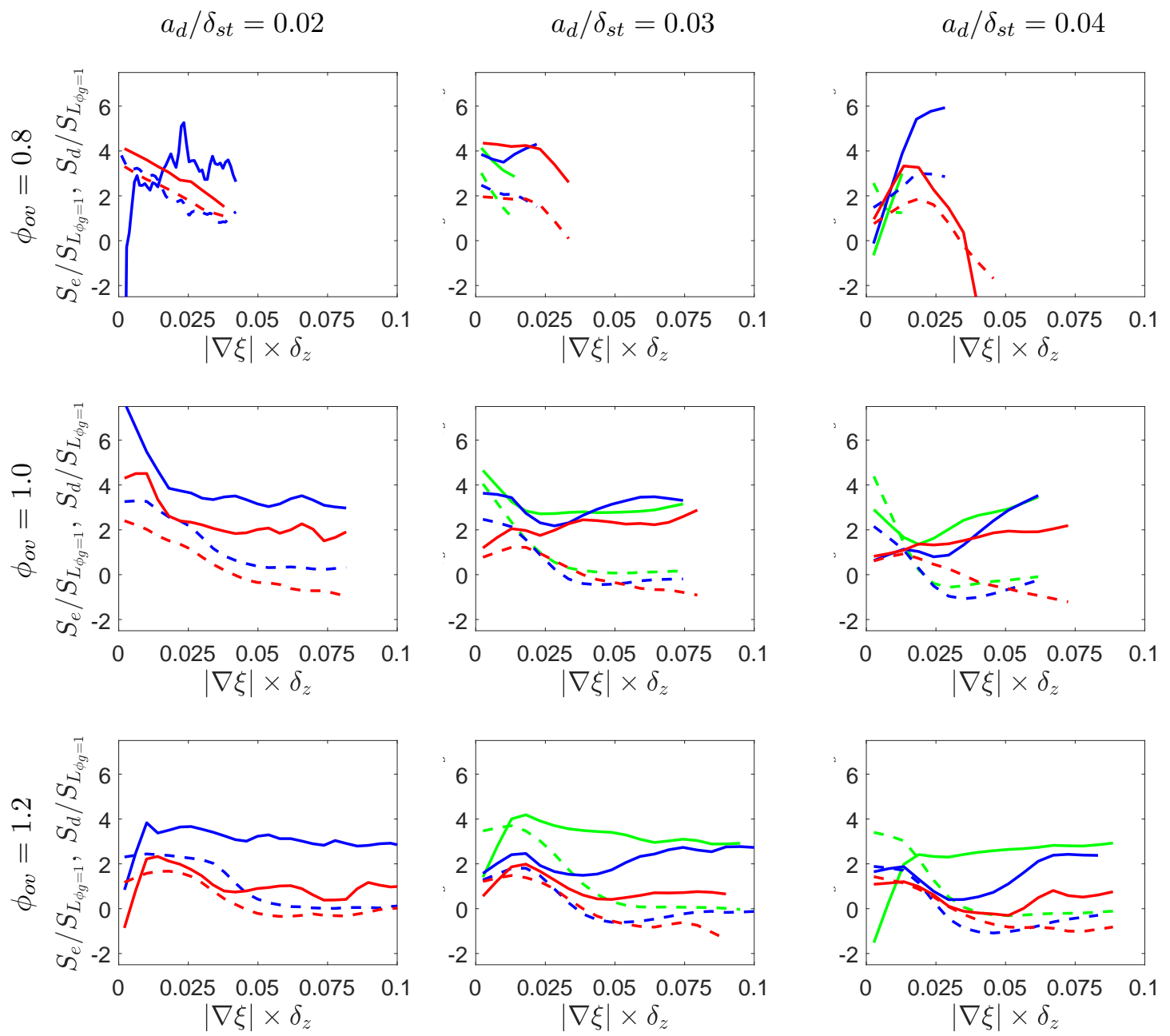

FIG. 17. Variations of the mean values of $S_{e} / S_{L_{\phi_{g}=1}}$ (solid line) and $S_{d} / S_{L_{\phi_{g}=1}}$ conditional on $|\nabla \xi| \times \delta_{z}$ for the samples corresponding to $0.1 \leq c \leq 0.9$ on the $\xi=\xi_{s t}$ isosurface for initial $a_{d} / \delta_{s t}=0.02,0.03$ and 0.04 (left to right) and overall equivalence ratios $\phi_{o v}=0.8,1.0$ and 1.2 (top to bottom), in the case of laminar (green), initial $u^{\prime} / S_{L_{\phi_{g}=1}}=4.0$ (blue) and initial $u^{\prime} / S_{L_{\phi_{g}=1}}=8.0$ (red) conditions.

From the above discussion, it becomes evident that the local curvature, strain rate and scalar gradient dependences of local edge flame speed for igniting droplet-laden mixtures remain qualitatively similar to the corresponding statistical dependences in the case of purely gaseous edge flame. This suggests that the modelling methodologies (e.g. Ref. ${ }^{62}$ ), which are usually employed for purely gaseous edge flames could potentially be extended for edge flames 
in droplet-laden mixtures. However, the models need to account for the effects of droplet size and number density (or overall equivalence ratio) along with the local curvature, strain rate and scalar gradient dependences of edge flame speed, which has significant implications on the predictions of flame stabilisation and lift-off height ${ }^{2425}$. Furthermore, in Large Eddy Simulations (LES), some of the observed local curvature, strain rate and scalar gradient dependences of the edge flame speed are expected to be observed in the resolved-scale ${ }^{49}$ but these behaviours are expected to be dependent on sub-grid scale modelling which need to account for the effects of droplet diameter and overall equivalence ratio (or number density) on turbulent edge flames in droplet-laden mixtures.

\section{CONCLUSIONS}

The effects of droplet diameter, overall (i.e. liquid+gaseous phases) equivalence ratio and turbulence intensity on the edge flame propagation statistics for localised forced ignition of uniformly dispersed n-heptane droplet-laden mixtures under decaying homogeneous isotropic turbulence have been analysed using three-dimensional DNS data. It has been found that the gaseous phase combustion takes place predominantly under fuel-lean mode even for globally stoichiometric (i.e. $\phi_{o v}=1.0$ ) and fuel-rich (e.g. $\phi_{o v}=1.2$ ) overall equivalence ratios. The tendency of fuel-lean combustion increases with increasing droplet size and decreasing overall equivalence ratio due to the slow evaporation of droplets, and reduced availability of gaseous fuel, respectively. The droplets have been found to induce flame wrinkling in the laminar cases but this effect is progressively eclipsed by the flame deformation due to fluid motion with increasing turbulence intensity. The most probable and mean values of the edge flame speed $S_{e}$ decrease from the theoretical laminar flame value $S_{L_{\phi_{g}=1}} \sqrt{\rho_{0} / \rho_{b}}$ in turbulent cases, and this deviation increases with increasing turbulence intensity. Furthermore, the probability of obtaining negative values of edge flame speed increases with increasing turbulence intensity. It has been found that both the initial droplet diameter $a_{d}$ and overall equivalence ratio $\phi_{o v}$ do not have significant influences on the qualitative distributions of $S_{e} / S_{L_{\phi_{g}=1}}$ for both laminar and turbulent flames. However, the droplet size and overall equivalence ratio have been shown to influence the gaseous phase composition, which along with droplet-induced flame deformation leads to a range of different values of $S_{e} / S_{L_{\phi_{g}=1}}$ in the laminar droplet cases. An increase in $a_{d}$ reduces the evaporation rate and 
thus the evaporated fuel in the gaseous phase mixes imperfectly with the surrounding air, which in turn gives rise to a greater likelihood of non-premixed mode of combustion. Thus, the typical edge flame structure becomes increasingly likely for large droplets and this tendency increases further with increasing $\phi_{o v}$ because of the larger number density of droplets. This, in turn, gives rise to a sharper peak of the PDFs of $S_{e} / S_{L_{\phi_{g}=1}}$ at $S_{e}^{0} / S_{L_{\phi_{g}=1}}=\sqrt{\rho_{0} / \rho_{b}}$ for larger droplet diameters and this trend strengthens further with increasing $\phi_{o v}$. It has been found that the displacement speed of the fuel mass fraction isosurface intersecting the stoichiometric mixture fraction isosurface remains the major contributor to the edge flame speed and that the displacement speed of the stoichiometric mixture fraction isosurface does not significantly affect the marginal PDF and strain rate and curvature dependences of the edge flame speed in this configuration. However, the displacement speed of the stoichiometric mixture fraction isosurface may have significant influence on the local scalar gradient (i.e. $\left|\nabla Y_{F}\right|$ and $|\nabla \xi|$ ) dependences of edge flame speed $S_{e}$ and this behaviour is particularly strong for large droplet cases where a relatively slow evaporation rate may induce strong local mixture inhomogeneity (i.e. large mixture fraction gradient).

The statistics of the displacement speed of the fuel mass fraction isosurface $S_{d}$ have been analysed further by examining its reaction, evaporation (due to the presence of droplets), normal and tangential diffusion components. It has been found that the magnitude of the evaporation component of displacement speed remains smaller than the magnitudes of reaction and normal diffusion components in the reaction zone $(0.5 \leq c \leq 0.9)$ for all cases considered here. This indicates that the evaporation of droplets does not affect the edge flame speed through the evaporation component of displacement speed. However, the composition of the reacting gaseous mixture is affected by the evaporation characteristics of the droplets, which eventually affects the local strain rate and scalar gradients and determines the growth rate of the hot gas kernel. The local edge flame speed has been found to be non-linearly dependent on the curvature of the fuel mass fraction isosurface. The tangential strain rate acting on the fuel mass fraction isosurface has been found to be mostly positively correlated but a negatively correlating branch has been observed for some cases. The edge flame speed has been found to exhibit non-monotonic dependences on the magnitudes of the gradients of both fuel mass fraction and mixture fraction. These curvature, strain rate and scalar gradient dependences of edge flame speed have been found to be qualitatively similar to the corresponding statistics reported for edge flames in purely gaseous 


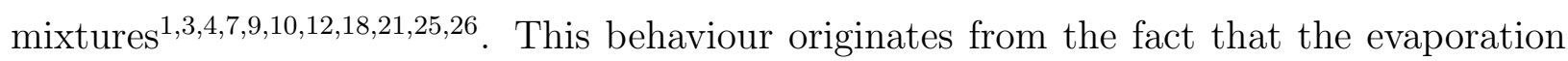
contribution to the edge flame speed remains negligible in comparison to the other components, which are also present for edge flames in purely gaseous phase mixtures. Furthermore, the strengths of the correlations of the edge flame speed with curvature and tangential strain rate have been found to be dependent on the droplet size and overall equivalence ratio, and the curvature and strain rate dependences of edge flame speed have been found to be weak for cases with large droplets.

The qualitative similarities of the edge flame statistics with those in the case of purely gaseous edge flames suggest that the modelling methodologies, which are usually employed for these edge flames could potentially be extended for edge flames in droplet-laden mixtures. However, the models need to include the effects of droplet size and number density (or overall equivalence ratio) along with the local curvature, strain rate and scalar gradient dependences of the edge flame speed in order to predict the edge flame propagation with high-fidelity. The modelling of edge flame propagation will be undertaken in future analyses. Finally, even though a number of previous analyses based on simple chemical mechanisms provided important insights into the edge flame propagation $\frac{1314|9| 12|24| 25}{4}$ and the findings from simple

chemistry based DNS ${ }^{11344}$ have been found to be qualitatively similar to those obtained from detailed chemistry simulations ${ }^{5}$, the findings of the current analysis need to be validated using detailed chemistry simulations for the sake of completeness, which also provides the basis for future investigations.

\section{ACKNOWLEDGEMENTS}

The financial support of the British Council, EPSRC, and National Education Ministry of the Turkish government and computational support of Rocket, Cirrus and ARCHER are gratefully acknowledged.

\section{REFERENCES}

${ }^{1}$ N. Chakraborty and E. Mastorakos, "Numerical investigation of edge flame propagation characteristics in turbulent mixing layers," Phys. Fluids 18, 1-18 (2006). 
${ }^{2}$ N. Chakraborty, E. Mastorakos, and R. S. Cant, "Effects of turbulence on spark ignition in inhomogeneous mixtures: A direct numerical simulation (DNS) study," Combust. Sci. Technol. 179, 293-317 (2007).

${ }^{3}$ H. Hesse, N. Chakraborty, and E. Mastorakos, "The effects of the Lewis number of the fuel on the displacement speed of edge flames in igniting turbulent mixing layers," Proc. Combust. Inst. 32, 1399-1407 (2009).

${ }^{4}$ N. Chakraborty, H. Hesse, and E. Mastorakos, "Numerical investigation of edge flame propagation behavior in an igniting turbulent planar jet," Combust. Sci. Technol. 182, 1747-1781 (2010).

${ }^{5}$ A. Neophytou, E. Mastorakos, and R. S. Cant, "DNS of spark ignition and edge flame propagation in turbulent droplet-laden mixing layers," Combust. Flame 157, 1071-1086 (2010).

${ }^{6}$ C. Turquand d'Auzay, V. Papapostolou, S. Ahmed, and N. Chakraborty, "Effects of turbulence intensity and biogas composition on the localized forced ignition of turbulent mixing layers," Combust. Sci. Technol. , 1-30 (2019).

${ }^{7} \mathrm{~S}$. H. Chung, "Stabilization, propagation and instability of tribrachial triple flames," Proc. Combust. Inst. 31, 877-892 (2007).

${ }^{8} \mathrm{P}$. Domingo and L. Vervisch, "Triple flames and partially premixed combustion in autoignition of non-premixed turbulent mixtures," Symp. Combust. 26, 233-240 (1996).

${ }^{9} \mathrm{~J}$. Dold, "Flame propagation in a nonuniform mixture: Analysis of a slowly varying Triple Flame," Combust. Flame 76, 71-88 (1989).

${ }^{10}$ L. J. Hartley and J. Dold, "Flame Propagation in a Nonuniform Mixture: Analysis of a Propagating Triple-Flame," Combust. Sci. Technol. 80, 23-46 (1991).

${ }^{11}$ G. R. Ruetsch, L. Vervisch, and A. Liñán, "Effects of heat release on triple flames," Phys. Fluids 7, 1447-1454 (1995).

${ }^{12}$ J. Buckmaster, "Edge-flames," Prog. Energy Combust. Sci. 28, 435-475 (2002),

${ }^{13}$ Y. S. Ko and S. H. Chung, "Propagation of unsteady tribrachial flames in laminar nonpremixed jets," Combust. Flame 118, 151-163 (1999).

${ }^{14} \mathrm{~K}$. Takita, S. Sakaguchi, and G. Masuya, "Premixed edge flame in a counterflow field with a stretch rate gradient," Combust. Flame 132, 343-351 (2003).

${ }^{15}$ K. Takita, M. Sado, G. Masuya, and S. Sakaguchi, "Experimental study of premixed single edge-flame in a counterflow field," Combust. Flame 136, 364-370 (2004). 
${ }^{16}$ S. H. Won, J. Kim, K. J. Hong, M. S. Cha, and S. H. Chung, "Stabilization mechanism of lifted flame edge in the near field of coflow jets for diluted methane," Proc. Combust. Inst. 30, 339-347 (2005),

${ }^{17}$ T. Echekki and J. H. Chen, "Structure and propagation of methanol-air triple flames," Combust. Flame 114, 231-245 (1998).

${ }^{18}$ H. G. Im and J. H. Chen, "Structure and propagation of triple flames in partially premixed hydrogen-air mixtures," Combust. Flame 119, 436-454 (1999).

${ }^{19}$ H. G. Im and J. H. Chen, "Effects of flow strain on triple flame propagation," Combust. Flame 126, 1384-1392 (2001).

${ }^{20}$ C. S. Yoo and H. G. Im, "Transient dynamics of edge flames in a laminar nonpremixed hydrogen-air counterflow," Proc. Combust. Inst. 30, 349-356 (2005).

${ }^{21}$ C. Pantano, "Direct simulation of non-premixed flame extinction in a methane - air jet with reduced chemistry," J. Fluid Mech. 514, 231-270 (2004).

${ }^{22} \mathrm{C}$. Jiménez and B. Cuenot, "DNS study of stabilization of turbulent triple flames by hot gases," Proc. Combust. Inst. 31, 1649-1656 (2007).

${ }^{23}$ C. Heeger, B. Böhm, S. F. Ahmed, R. Gordon, I. Boxx, W. Meier, A. Dreizler, and E. Mastorakos, "Statistics of relative and absolute velocities of turbulent non-premixed edge flames following spark ignition," Proc. Combust. Inst. 32, 2957-2964 (2009).

${ }^{24}$ S. Karami, E. R. Hawkes, M. Talei, and J. H. Chen, "Mechanisms of flame stabilisation at low lifted height in a turbulent lifted slot-jet flame," J. Fluid Mech. 777, 633-689 (2015).

${ }^{25}$ S. Karami, E. R. Hawkes, M. Talei, and J. H. Chen, "Edge flame structure in a turbulent lifted flame: A direct numerical simulation study," Combust. Flame 169, 110-128 (2016).

${ }^{26}$ A. Krisman, E. R. Hawkes, and J. H. Chen, "Two-stage autoignition and edge flames in a high pressure turbulent jet," J. Fluid Mech. 824, 5-41 (2017).

${ }^{27}$ J. Greenberg, Y. Mindelis, and M. Matalon, "Edge Flames with a Fuel Spray and Reactants Having Different Diffusivities," in 48th AIAA Aerosp. Sci. Meet. Incl. New Horizons Forum Aerosp. Expo., January (American Institute of Aeronautics and Astronautics, Reston, Virigina, 2010) pp. 1-12.

${ }^{28}$ J. B. Greenberg, "Droplet size distribution effects in an edge flame with a fuel spray," Combust. Flame 179, 228-237 (2017).

${ }^{29}$ E. Fernández-Tarrazo, A. L. Sánchez, A. Liñán, and F. A. Williams, "A simple one-step chemistry model for partially premixed hydrocarbon combustion," Combust. Flame 147, 


\section{$32-38$ (2006).}

${ }^{30}$ Y. Haruki, A. L. Pillai, T. Kitano, and R. Kurose, "Numerical investigation of flame propagation in fuel droplet arrays," Atomization and Sprays 28, 357-388 (2018).

${ }^{31}$ J. Réveillon and L. Vervisch, "Spray vaporization in nonpremixed turbulent combustion modeling: a single droplet model," Combust. Flame 121, 75-90 (2000).

${ }^{32}$ A. P. Wandel, N. Chakraborty, and E. Mastorakos, "Direct numerical simulations of turbulent flame expansion in fine sprays," Proc. Combust. Inst. 32 II, 2283-2290 (2009).

${ }^{33}$ C. Vázquez-Espí and A. Liñán, "Fast, non-diffusive ignition of a gaseous reacting mixture subject to a point energy source," Combust. Theory Model. 5, 485-498 (2001).

${ }^{34}$ C. Vázquez-Espí and A. Liñán, "Thermal-diffusive ignition and flame initiation by a local energy source," Combust. Theory Model. 6, 297-315 (2002).

${ }^{35}$ D. R. Ballal and A. H. Lefebvre, "Spark ignition of turbulent flowing gases," in 15th Aerosp. Sci. Meet., Vol. 155 (American Institute of Aeronautics and Astronautics, Reston, Virginia, 1977) pp. 129-155.

${ }^{36}$ T. Poinsot and S. Lele, "Boundary conditions for direct simulations of compressible viscous flows," J. Comput. Phys. 101, 104-129 (1992).

${ }^{37}$ A. Wray, "Minimal storage time advancement schemes for spectral methods," (1990), NASA Ames Research Center, California.

${ }^{38}$ R. S. Rogallo, "Numerical experiments in homogeneous turbulence," Tech. Rep. (NASA Ames, 1981).

${ }^{39}$ M. Nakamura, F. Akamatsu, R. Kurose, and M. Katsuki, "Combustion mechanism of liquid fuel spray in a gaseous flame," Phys. Fluids 17, 1-14 (2005).

${ }^{40} \mathrm{Y}$. Wang and C. J. Rutland, "Effects of temperature and equivalence ratio on the ignition of n-heptane fuel spray in turbulent flow," Proc. Combust. Inst. 30, 893-900 (2005).

${ }^{41}$ H. Watanabe, R. Kurose, S.-M. Hwang, and F. Akamatsu, "Characteristics of flamelets in spray flames formed in a laminar counterflow," Combust. Flame 148, 234-248 (2007).

${ }^{42} \mathrm{~S}$. Sreedhara and K. Y. Huh, "Conditional statistics of nonreacting and reacting sprays in turbulent flows by direct numerical simulation," Proc. Combust. Inst. 31, 2335-2342 (2007).

${ }^{43}$ J. Xia and K. H. Luo, "Direct numerical simulation of inert droplet effects on scalar dissipation rate in turbulent reacting and non-reacting shear layers," Flow, Turbul. Combust. 84, 397-422 (2010) 
${ }^{44}$ A. Fujita, H. Watanabe, R. Kurose, and S. Komori, "Two-dimensional direct numerical simulation of spray flames - Part 1: Effects of equivalence ratio, fuel droplet size and radiation, and validity of flamelet model," Fuel 104, 515-525 (2013).

${ }^{45}$ A. P. Wandel, "Extinction predictors in turbulent sprays," Proc. Combust. Inst. 34, 1625$1632(2013)$.

${ }^{46}$ A. P. Wandel, "Influence of scalar dissipation on flame success in turbulent sprays with spark ignition," Combust. Flame 161, 2579-2600 (2014).

${ }^{47}$ G. Ozel Erol, J. Hasslberger, M. Klein, and N. Chakraborty, "A direct numerical simulation analysis of spherically expanding turbulent flames in fuel droplet-mists for an overall equivalence ratio of unity," Phys. Fluids 30, 086104 (2018).

${ }^{48}$ G. Ozel Erol, J. Hasslberger, M. Klein, and N. Chakraborty, "A direct numerical simulation investigation of spherically expanding flames propagating in fuel droplet-mists for different droplet diameters and overall equivalence ratios," Combust. Sci. Technol. 30, 1-35 (2019).

${ }^{49} \mathrm{Y}$. Hu and R. Kurose, "Nonpremixed and premixed flamelets LES of partially premixed spray flames using a two-phase transport equation of progress variable," Combust. Flame 188, 227-242 (2018).

${ }^{50}$ C. Turquand d'Auzay, V. Papapostolou, S. F. Ahmed, and N. Chakraborty, "On the minimum ignition energy and its transition in the localised forced ignition of turbulent homogeneous mixtures," Combust. Flame 201, 104-117 (2019).

${ }^{51}$ R. Yu and X.-S. Bai, "Direct numerical simulation of lean hydrogen/air auto-ignition in a constant volume enclosure," Combust. Flame 160, 1706-1716 (2013).

${ }^{52}$ C. Pera, S. Chevillard, and J. Reveillon, "Effects of residual burnt gas heterogeneity on early flame propagation and on cyclic variability in spark-ignited engines," Combust. Flame 160, 1020-1032 (2013).

${ }^{53}$ S. Hayashi, S. Kumagai, and T. Sakai, "Propagation velocity and structure of flames in droplet-vapor-air mixtures," Combust. Sci. Technol. 15, 169-177 (1977).

${ }^{54} \mathrm{M}$. Lawes and A. Saat, "Burning rates of turbulent iso-octane aerosol mixtures in spherical flame explosions," Proc. Combust. Inst. 33, 2047-2054 (2011).

${ }^{55}$ A. M. Briones, S. K. Aggarwal, and V. R. Katta, "A numerical investigation of flame lift off, stabilization, and blowout," Phys. Fluids 18, 1-13 (2006). 
${ }^{56}$ K. Jenkins, M. Klein, N. Chakraborty, and R. S. Cant, "Effects of strain rate and curvature on the propagation of a spherical flame kernel in the thin-reaction-zones regime," Combust. Flame 145, 415-434 (2006).

${ }^{57}$ M. Klein, N. Chakraborty, K. Jenkins, and R. S. Cant, "Effects of initial radius on the propagation of premixed flame kernels in a turbulent environment," Phys. Fluids 18, $055102(2006)$.

${ }^{58}$ N. Chakraborty, M. Klein, and R. S. Cant, "Stretch rate effects on displacement speed in turbulent premixed flame kernels in the thin reaction zones regime," Proc. Combust. Inst. 31, 1385-1392 (2007).

${ }^{59}$ N. Chakraborty, M. Klein, and R. S. Cant, "Effects of turbulent reynolds number on the displacement speed statistics in the thin reaction zones regime of turbulent premixed combustion," J. Combust. 2011, 1-19 (2011).

${ }^{60}$ J. H. Chen and H. G. Im, "Correlation of flame speed with stretch in turbulent premixed methane/air flames," Symp. Combust. 27, 819-826 (1998).

${ }^{61}$ G. V. Nivarti and R. S. Cant, "Stretch rate and displacement speed correlations for increasingly-turbulent premixed flames," Flow, Turbul. Combust. 102, 957-971 (2019).

${ }^{62}$ C. Müller, H. Breitbach, and N. Peters, "Partially premixed turbulent flame propagation in jet flames," Symp. Combust. 25, 1099-1106 (1994). 